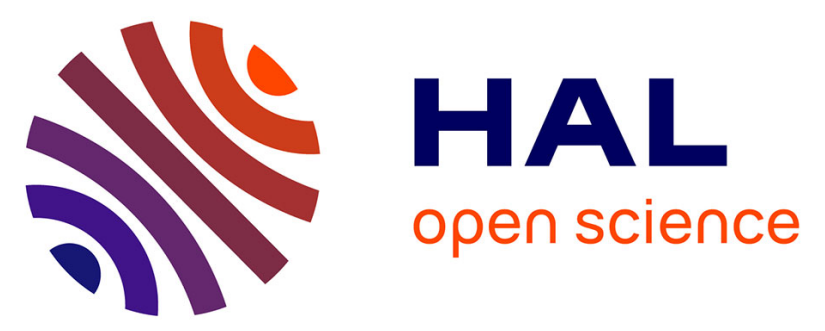

\title{
Argon K L L Auger spectrum: Initial states, core-hole lifetimes, shake, and knock-down processes
}

Ralph Püttner, Philippe Holzhey, Mateja Hrast, Matjaz Žitnik, Gildas

Goldsztejn, Tatiana Marchenko, Renaud Guillemin, Loïc Journel, Dimitris

Koulentianos, Oksana Travnikova, et al.

\section{To cite this version:}

Ralph Püttner, Philippe Holzhey, Mateja Hrast, Matjaz Žitnik, Gildas Goldsztejn, et al.. Argon K L L Auger spectrum: Initial states, core-hole lifetimes, shake, and knock-down processes. Physical Review A, 2020, 102 (5), 10.1103/PhysRevA.102.052832 . hal-03439162

\section{HAL Id: hal-03439162 https://hal.science/hal-03439162}

Submitted on 22 Nov 2021

HAL is a multi-disciplinary open access archive for the deposit and dissemination of scientific research documents, whether they are published or not. The documents may come from teaching and research institutions in France or abroad, or from public or private research centers.
L'archive ouverte pluridisciplinaire HAL, est destinée au dépôt et à la diffusion de documents scientifiques de niveau recherche, publiés ou non, émanant des établissements d'enseignement et de recherche français ou étrangers, des laboratoires publics ou privés. 


\section{Freie Universität}

Title:

\section{Argon KLL Auger spectrum: Initial states, core-hole lifetimes, shake, and knock-down processes}

Author(s): Ralph Püttner, Philippe Holzhey, Mateja Hrast, Matjaz Žitnik, Gildas Goldsztejn, Tatiana Marchenko, Renaud Guillemin, Loïc Journel, Dimitris Koulentianos, Oksana Travnikova, Moustafa Zmerli, Denis Céolin, Yoshiro Azuma, Satoshi Kosugi,

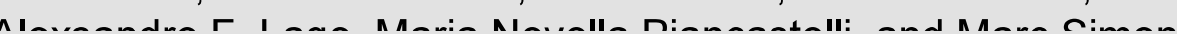

Document type: Preprint

Terms of Use: $\quad$ Copyright applies. A non-exclusive, non-transferable and limited right to use is granted. This document is intended solely for personal, non-commercial use.

Citation:

"Argon KLL Auger spectrum: Initial states, core-hole lifetimes, shake, and knock-down processes

Ralph PüttnerPhys. Rev. A 102, 052832 ; https://doi.org/10.1103/PhysRevA.102.052832" 


\section{Argon $K L L$ Auger spectrum:}

\section{Initial states, core-hole lifetimes, shake and knock-down processes}

Ralph Püttner, ${ }^{1, *}$ Philippe Holzhey, ${ }^{1, \dagger}$ Mateja Hrast, ${ }^{2}$ Matjaz Žitnik, ${ }^{2,3}$

Gildas Goldsztejn, ${ }^{4,5}$ Tatiana Marchenko, ${ }^{4,6}$ Renaud Guillemin, ${ }^{4,6}$

Loïc Journel, ${ }^{4,6}$ Dimitris Koulentianos, ${ }^{7,4, \ddagger}$ Oksana Travnikova, ${ }^{4,6}$

Moustafa Zmerli, ${ }^{4}$ Denis Céolin, ${ }^{6}$ Yoshiro Azuma, ${ }^{8}$ Satoshi Kosugi, ${ }^{8}$

Alexsandre F. Lago, ${ }^{9}$ Maria Novella Piancastelli, ${ }^{4,10}$ and Marc Simon ${ }^{4,6}$

${ }^{1}$ Fachbereich Physik, Freie Universität Berlin,

Arnimallee 14, D-14195 Berlin, Germany

${ }^{2}$ J. Stefan Institute, Jamova 39, SI-1000 Ljubljana, Slovenia

${ }^{3}$ Faculty of Mathematics and Physics, University of Ljubljana,

Jadranska 19, SI-1000 Ljubljana, Slovenia

${ }_{4}^{4}$ Sorbonne Université, CNRS, Laboratoire de Chimie Physique-Matière et Rayonnement,

LCPMR, F-75005 Paris Cedex 05, France

${ }^{5}$ Université Paris-Saclay, CNRS, Institut des

Sciences Moléculaires d'Orsay, 91405, Orsay, France

${ }^{6}$ Synchrotron SOLEIL, L'Orme des Merisiers,

Saint-Aubin, F-91192 Gif-sur-Yvette Cedex, France

${ }^{7}$ Department of Physics, University of Gothenburg,

Origovägen 6B, SE-412 96 Gothenburg, Sweden

${ }^{8}$ Department of Materials and Life Sciences,

Sophia University, Tokyo 102-8554, Japan

${ }^{9}$ Centro de Ciências Naturais e Humanas,

Universidade Federal do ABC, 09210-580, Santo André-SP, Brazil

${ }^{10}$ Department of Physics and Astronomy,

Uppsala University, SE-75120 Uppsala, Sweden

(Dated: March 18, 2021) 


\begin{abstract}
State-of-the-art Argon KLL Auger spectra measured using photon energies of $h \nu=3216 \mathrm{eV}$ and $3400 \mathrm{eV}$ are presented along with an $\mathrm{Ar}[1 s]$ photoelectron spectrum (squared brackets indicate holes in the respective orbital). The two different photon energies used for measuring the Auger spectra allow distinguishing between the shake transitions during the Auger decay and the Auger transitions of the photoelectron satellites. A complete assignment of satellite transitions is provided, partially baseed on configuration interaction calculations. In addition, Ar $[1 s 3(s, p)] n^{\prime} l^{\prime} \rightarrow\left[2 p^{2}\left({ }^{1} D_{2}\right)\right]$ transitions are observed, which can be explained by knock-down transitions leading to a direct exchange of angular momenta between the excited electron and the Auger electron. The lifetime broadenings of the Ar $[2 s]$ single core-hole state and the $\left[2 s^{2}\right]$ as well as $[2 s 2 p]$ double core-hole states are also determined, confirming previously observed trends for double core-hole states.
\end{abstract}

PACS numbers: 33.15.Ry, 33.60.+q, 33.80.Eh, 33.20.Rm

*Electronic address: puettner@physik.fu-berlin.de

${ }^{\dagger}$ present address: Clarendon Laboratory, Department of Physics, University of Oxford, Oxford, OX1 3PU, UK

${ }^{\ddagger}$ present address: Chemical Sciences and Engineering Division, Argonne National Laboratory, 9700 S Cass Avenue, Lemont, IL 60439, USA 


\section{INTRODUCTION}

The KLL Auger spectrum of argon has been studied extensively in the 70's and 80's of the last century, both theoretically $[1-4]$ and experimentally $[5,6]$. In those studies, the dominating diagram lines $[1 s] \rightarrow\left[2 s^{2}\left({ }^{1} S\right)\right],[1 s] \rightarrow\left[2 s 2 p\left({ }^{1} P,{ }^{3} P\right)\right]$, and $[1 s] \rightarrow\left[2 p^{2}\left({ }^{1} S,{ }^{1} D,{ }^{3} P\right)\right]$ were unambiguously assigned; note that throughout this publication squared brackets indicate holes in the respective orbital. Moreover, the experimental studies also reported a number of satellite structures. For the $K L L$ Auger spectrum of argon such satellites are due to transitions of valence electrons into unoccupied orbitals. These transitions accompany the photoionization as well as the Auger decay and are caused by two different processes, namely shake and knock. Shake transitions are a consequence of the relaxation process subsequent to ionization which causes a change in the radial wavefunctions of the orbitals. As a consequence of the ionization process, the orbitals before and after ionization are not orthogonal to each other and the shake transitions can be described by the matrix elements $\left|\left\langle n l \mid n^{\prime} l^{\prime}, \epsilon^{\prime} l^{\prime}\right\rangle\right|^{2} \neq 0$. Here $l$ indicates the orbital angular momentum, $n$ the orbital quantum number, and $\epsilon$ a continuum wavefunction. Moreover, prime indicates an originally unoccupied orbital in its relaxed form due to ionization and no prime an occupied orbital in the atomic ground state. The matrix element allows only monopole transitions, i.e. $l=l^{\prime}$ and in case of $\operatorname{Ar} 1 s$ photoionization only ${ }^{2} S$ final states for the ion, e.g. $\left[1 s 3 p\left({ }^{1,3} P\right)\right] 4 p\left({ }^{2} S\right)$. Note that throughout this publication we omit prime and double prime (see below) when the principle quantum number $n$ is specified (here $n^{\prime}=4$ ).

In a knock process the outgoing electron interacts via Coulomb repulsion with an electron from the valence shell. This allows the exchange of energy and angular momentum between the two electrons and may lead e.g. in photoionization to the final states $\left[1 s 3 p\left({ }^{1,3} P\right)\right] 4 s\left({ }^{2} P\right)$. This semi-classical picture is described in a fully quantum mechanical treatment by configuration interaction between the outgoing electron and the electrons remaining in the ion [7]. In the photoionization process one can observe $n^{\prime}=n$, i.e. the absence of shake which causes the main line as well as $n^{\prime}>n$, which causes the shake-up or knock-up satellites.

In case of the Auger decay the shake process can be described with the matrix element $\left|\left\langle n^{\prime} l^{\prime} \mid n^{\prime \prime} l^{\prime \prime}, \epsilon^{\prime \prime} l^{\prime \prime}\right\rangle\right|^{2}$. Here double prime indicate indicates an originally unoccupied orbital in its relaxed form for the case of a double core hole (DCH). As in case of the photoionization, 
an additional electron can be promoted to a higher shell $\left(n^{\prime \prime}>n^{\prime}\right)$ or to the continuum $\epsilon$, which is called shake up/knock up and shake off/knock off, respectively. The Auger process also allows $n^{\prime \prime}=n^{\prime}$, which is in case of argon for $n^{\prime}=3$ a diagramm line and for $n^{\prime} \geq 4$ a spectator decay. Finally, in the Auger decay of a photoelectron satellite $n^{\prime \prime}<n^{\prime}$ can be found; this describes a shake-down/knock-down process.

Outgoing electrons with high kinetic energy can interact with the valence electrons only within a short time interval leading to a negligible exchange of energy and momentum. These conditions are known as sudden limit and allow only shake transitions [7]. The fact that different photon energies lead only to small changes in the satellite structure of the photoelectron spectra suggests that for the formation of satellite structures the shake processes are normally dominant over the knock processes [8]. Moreover, for a large number of satellite structures one cannot distinguish between shake and knock based on the given quantum numbers. Because of this all transitions which are assumed to be predominantly due to the shake process are labeled as such, although knock contributions cannot be excluded. However, some transitions are explicitly assumed to be dominated by the knock process and are, therefore, labeled in this way. Note that a more detailed recent discussion of the different shake and knock processes has been given by Travnikova et al. [9].

We present here a comprehensive assignment of all satellite structures in the $K L L$ Auger decay after argon $1 s$ photoionization. Despite the complexity of the Auger spectrum, we are able to identify up to six types of different processes (see below for a detailed list).

To perform such a task, both the ionization process and the Auger decay have to be taken into account. In principle both, the ionization and the decay can be accompanied by a shake-up/knock-up or a shake-off/knock-off process resulting in rich satellite structures. Fig. 1 shows the relevant processes for this work, namely shake-free/knock-free or also labeled direct transitions (green solid arrows), shake-up/knock-up transitions (blue dash-dotted arrows), shake-down/knock-down transitions (red dotted arrows), and shakeoff/knock-off transitions (magenta dashed arrows); since we measure single-electron emission the latter transitions are not directly visible in the present spectra, but they influence the Auger spectrum by their decay. By taking into account direct as well as shake and knock transitions in both, the ionization and the Auger decay, we can distinguish six types of Auger transitions, which are indicated in Fig. 1 by numbers below the down-arrows. For convenience, their properties are summarized in the following enumeration. 
Type 1: Direct transition in both ionisation and decay, i.e. $[1 s] \rightarrow\left[2 p^{2}\right]$ transitions. These transtions are also known as diagram lines.

Type 2: Direct transition in the ionisation process and shake-up during the Auger decay, i.e. $[1 s] \rightarrow\left[2 p^{2} 3 l\right] n^{\prime \prime} l^{\prime \prime}$.

Type 3: Shake-up during the ionisation process and shake-down/knock-down during the Auger decay, i.e. $[1 s 3 l] n l^{\prime} \rightarrow\left[2 p^{2}\right]$. We shall show below that this type of Auger transitions is mostly due to the knock-mechanism.

Type 4: Shake-up during the ionisation process and direct transition during Auger decay, i.e. $[1 s 3 l] n^{\prime} l^{\prime} \rightarrow\left[2 p^{2} 3 l\right] n^{\prime \prime} l^{\prime \prime}$ with $n^{\prime}=n^{\prime \prime}$ and $l^{\prime}=l^{\prime \prime}$. These transitions are also known as spectator Auger decay.

Type 5: Shake-up during both ionisation and decay, i.e. $[1 s 3 l] n^{\prime} l^{\prime} \rightarrow\left[2 p^{2} 3 l\right] n^{+\prime \prime} l^{\prime \prime}$ with $n^{+}>n$

Type 6: Shake off during ionization and direct transition during Auger decay, i.e. $[1 s 3 l] \rightarrow\left[2 p^{2} 3 l\right]$

Despite the fact that all processes are possible, in Refs. $[5,6]$ the satellites were discussed only in terms of Auger decays of the shake-up (type 4) or shake-off (type 6) satellites of the photoionization process. In the cited publications $[5,6]$ the valence shell photoionization satellites, i.e. $[1 s 3(s, p)] n^{\prime} l^{\prime}, \epsilon^{\prime} l^{\prime}$ states, and core-level photoionization satellites, i.e. $[1 s 2(s, p)] n^{\prime} l^{\prime}$ states, were taken into account as initial states of the Auger decay. However, during the decay only two-electron direct Auger transitions (green solid down arrows of type 1, 4, and 6) were considered, i.e. shake processes during the Auger decay (type 2,3, and 5) were neglected in the assignment of the spectral features. Moreover, in 1997 Volker Schmidt stated in his monograph [10] that shake processes during the Auger decay were not observed at that time. For the molecules isoelectronic to argon, namely $\mathrm{HCl}, \mathrm{H}_{2} \mathrm{~S}, \mathrm{PH}_{3}$, and $\mathrm{SiH}_{4}$, similar assignments of the different structures visible in the $K L L$ Auger spectra are given, see Refs. $[5,11,12]$.

In resonant $K L L$ spectra shake transitions in the valence shell during Auger decay (type 5) are actually common [13-17], since the excited species possess an open-shell structure. Nevertheless, they are widely neglected in the analysis of normal Auger spectra and were found only for the open-shell atoms sodium [18] and silicon [19] as well as by some of the present authors for the closed-shell molecule $\mathrm{H}_{2} \mathrm{~S}$ [20]. In addition, this study on $\mathrm{H}_{2} \mathrm{~S}$ showed Auger decays of the S $[1 s \mathrm{Val}] n^{\prime} l^{\prime}$ ( $\mathrm{Val}$ : valence orbital) shake-up states of photoion- 


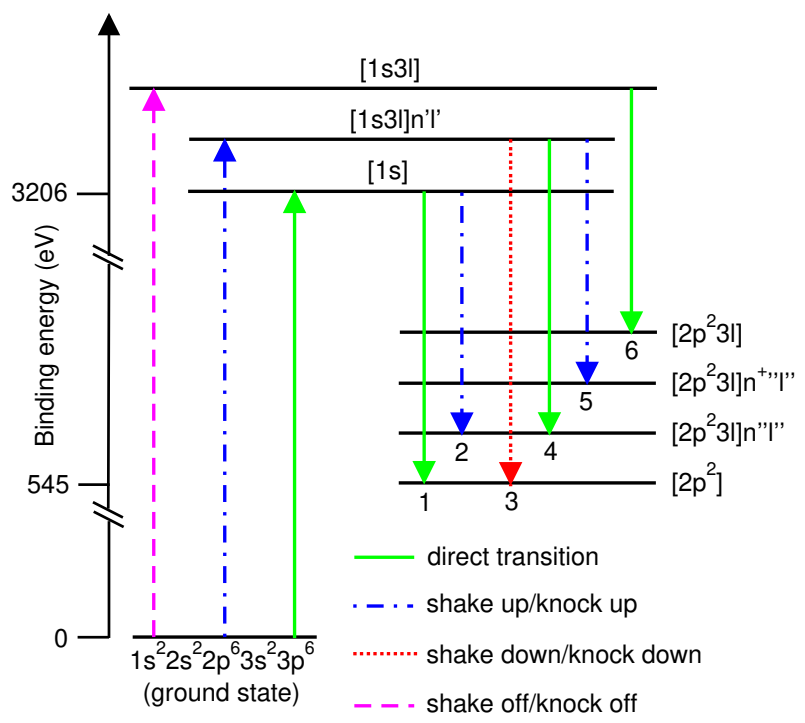

FIG. 1: (color online) Schematic picture of the ionization and the decay processes contributing to the $K L L$ Auger spectrum of argon. The green solid lines indicate shake-free/knock-free direct transitions, while the blue dash-dotted lines and red dotted lines represent shake-up/knock-up and shake-down/knock-down processes, respectively. The dashed magenta line indicates a double ionization process based on the shake-off/knock-off mechanism which is not observed directly but contributes to the Auger spectrum. Note that $n^{+}>n$, i.e. $n^{\prime} l^{\prime} \rightarrow n^{+\prime \prime} l^{\prime \prime}$ describes a shakeup/knock-up process (type 5). The different types of Auger processes are labeled by numbers below the down-arrows. For details, see text.

ization process. Auger decays of the $[1 s 2(s, p)] n^{\prime} l^{\prime}$ core level shake-up states were not clearly identified although they were used in earlier publications for the assignment of the satellites in the $K L L$ Auger spectrum of $\mathrm{H}_{2} \mathrm{~S}[11,12]$ and argon [6]. In Ref. [20] the different processes are identified by the photon energy used for the photoionization process; such a procedure became possible in recent years due to significant improvement in instrumentation for hard X-ray photoelectron spectroscopy (HAXPES) also available for gas-phase measurements [21]. In more detail, a shake process during the Auger decay (type 2) is possible at all photon energies above the $[1 s]$ ionization energy. Contrary to this, Auger decays of the $[1 s$ Val $] n^{\prime} l^{\prime}$ and $[1 s 2(s, p)] n^{\prime} l^{\prime}$ shake states (types 4 to 6 ) are possible only with photon energies that are high enough to form the corresponding initial state, i.e. $h \nu>E_{b i n}(1 s)+E_{\text {exc }}\left(3 l \rightarrow n^{\prime} l^{\prime}\right)$ with $E_{\text {bin }}(1 s)$ being the Ar $1 s$ binding energy and $E_{\text {exc }}\left(3 l \rightarrow n^{\prime} l^{\prime}\right)$ the excitation energy for a $3 l$ electron into the $n^{\prime} l^{\prime}$ level. 
The present study of the $K L L$ Auger spectrum of argon is performed by using the same approach as in Ref. [20], i.e. by using different ionization energies, and found likewise shake processes during the Auger decay (type 2) as well as Auger decays of $[1 s 3(s, p)] n^{\prime} l^{\prime}$ satellite states in the photoionization process (type 4 to 6 ). These results are fully confirmed by complementary calculations. For a better understanding of the Auger decays of $[1 s 3(s, p)] n^{\prime} l^{\prime}$ satellites, we also present and discuss the satellites in the $\operatorname{Ar}[1 s]$ photoelectron spectrum.

Although the present results are similar to those observed for $\mathrm{H}_{2} \mathrm{~S}$ [20], the spectral structure of argon is, however, simpler than that of $\mathrm{H}_{2} \mathrm{~S}$, due to the absence of the nuclear degrees of freedom, so that the electronic structure could be identified in deeper detail. In particular, in this way we obtained access to the highly excited double core-hole states of the configurations $\left[2 p^{2} 3 p\right] n^{\prime \prime}\left(p^{\prime \prime}, d^{\prime \prime}\right)$ and observed the splitting of the $\left[2 p^{2}\left({ }^{1} D\right) 3 p\right]$ configuration into the components $\left({ }^{2} P,{ }^{2} D,{ }^{2} F\right)$ due to the coupling of the $[3 p]$ hole to the $\left[2 p^{2}\left({ }^{1} D\right)\right]$ holes. In addition, $[1 s 3(s, p)] n^{\prime}\left(s^{\prime}, p^{\prime}\right) \rightarrow\left[2 p^{2}\left({ }^{1} D\right)\right]$ transitions are observed, which are predominantly due to knock-down processes (type 3). Moreover, the three components of the total angular momentum $J$ to the final states ${ }^{3} P_{2},{ }^{3} P_{1}$, and ${ }^{3} P_{0}$ of the $[1 s] \rightarrow\left[2 s 2 p\left({ }^{3} P\right)\right]$ diagram lines are resolved and for all diagram lines accurate energies are reported. Finally, lifetime broadenings of the $[2 s]$ single core-hole state $(\mathrm{SCH})$ and the $\left[2 s^{2}\right]$ as well $[2 s 2 p]$ double core-hole (DCH) states are given, confirming recent results which demonstrated that lifetime broadenings of DCH states are not just given by the sum of those ones of the involved SCH states [22-24]

\section{EXPERIMENTAL SETUP AND CALCULATIONAL DETAILS}

The measurements were performed at the SOLEIL synchrotron, France, on the GALAXIES beamline, with an endstation dedicated to HAXPES [21, 25]. Linearly polarized light was provided by a U20 undulator and energy selected by a Si(111) doublecrystal monochromator. Photoelectron spectra were collected with a SCIENTA EW4000 hemispherical electron analyzer specifically designed for HAXPES.

The $K L_{2,3} L_{2,3}$ Auger spectra relevant for the present study were measured using the photon energies $h \nu=3216 \mathrm{eV}$ and $3400 \mathrm{eV}$ in order to discriminate different processes like Auger transitions of the photoelectron satellites and shake transitions during the Auger decay; for details see below. Moreover, a $K L L$ overview spectrum including final states 
with $L_{1}$ holes has been measured at a photon energy of $4500 \mathrm{eV}$. For these measurements an analyser pass energy of $E_{\text {pass }}=200 \mathrm{eV}$ and a slit width of $500 \mu \mathrm{m}$ was applied, resulting in a spectrometer resolution of $\cong 550 \mathrm{meV}$. The Ar $[1 s]$ photoelectron spectrum was recorded using the same parameter settings for the electron analyser. However, the additional photon bandwidth at the used photon energy $h \nu=3900 \mathrm{eV}$ leads to a larger total resolution of $\cong 700$ meV. For the Ar $[2 s]$ photoelectron spectrum a photon energy of $2400 \mathrm{eV}$, a slit width of 300 $\mu \mathrm{m}$ and a pass energy of $E_{\text {pass }}=200 \mathrm{eV}$ were used, giving a Gaussian resolution of 545(10) meV.

The $\operatorname{Ar}[1 s]$ photoelectron spectrum was calibrated to the value of $E_{b i n}=3206.3(3)$ $\mathrm{eV}$ for the binding energy [26] while for the Auger spectra a kinetic energy of 2660.51(1) eV for the $[1 s] \rightarrow\left[2 p^{2}\left({ }^{1} D_{2}\right)\right]$ transition was used [6]. The Ar $[2 s]$ photoelectron spectrum was calibrated using a small $\mathrm{N}_{2}$ impurity with binding energies for the $\mathrm{N}\left[1 s_{g}\right]$ and $\mathrm{N}\left[1 s_{u}\right]$ core hole states of 409.93(1) eV and 409.82(1) eV, respectively [27].

The theoretical approach based on configuration interaction reproduced recently the measured Ar KLL spectrum [22]. The same semi-relativistic approach is employed below to estimate a spectral contribution of the shake-up Auger $K M-L L M$ decay. In parallel, a fully relativistic multi-configuration Dirac-Fock (MCDF) calculation of Auger amplitude between relaxed initial and final states was executed [28, 29], as described in detail in a recent study performed by some of the present authors [30]. In both cases the results are compared to experimental data using final states described by a mixture of $\left[2 p^{2} 3 p\right](3 d, 4 s, 4 p, 4 d, 5 p, 6 p)$ configurations.

\section{THE Ar $[1 s]$ PHOTOELECTRON SPECTRUM}

We first discuss the Ar $[1 s]$ photoelectron spectrum including the shake-up structures originating from valence orbitals, as such shake processes are relevant for about $20 \%$ of the transitions and produce additional initial states for the Auger decay [31-33]. The valence shake transitions of Ar have been investigated theoretically by Dyall [31] and experimentally by Southworth et al. [8]. Their work focused on the single shake-up transitions to the $[1 s 3 p] n^{\prime} p^{\prime}$ and $[1 s 3 s] n^{\prime} s^{\prime}$ final states and demonstrated that the calculations of Dyall [31] describe the single shake-up region in terms of energy positions and intensities very well. We present in Fig. 2 a spectrum with improved signal-to-noise ratio and high resolution 


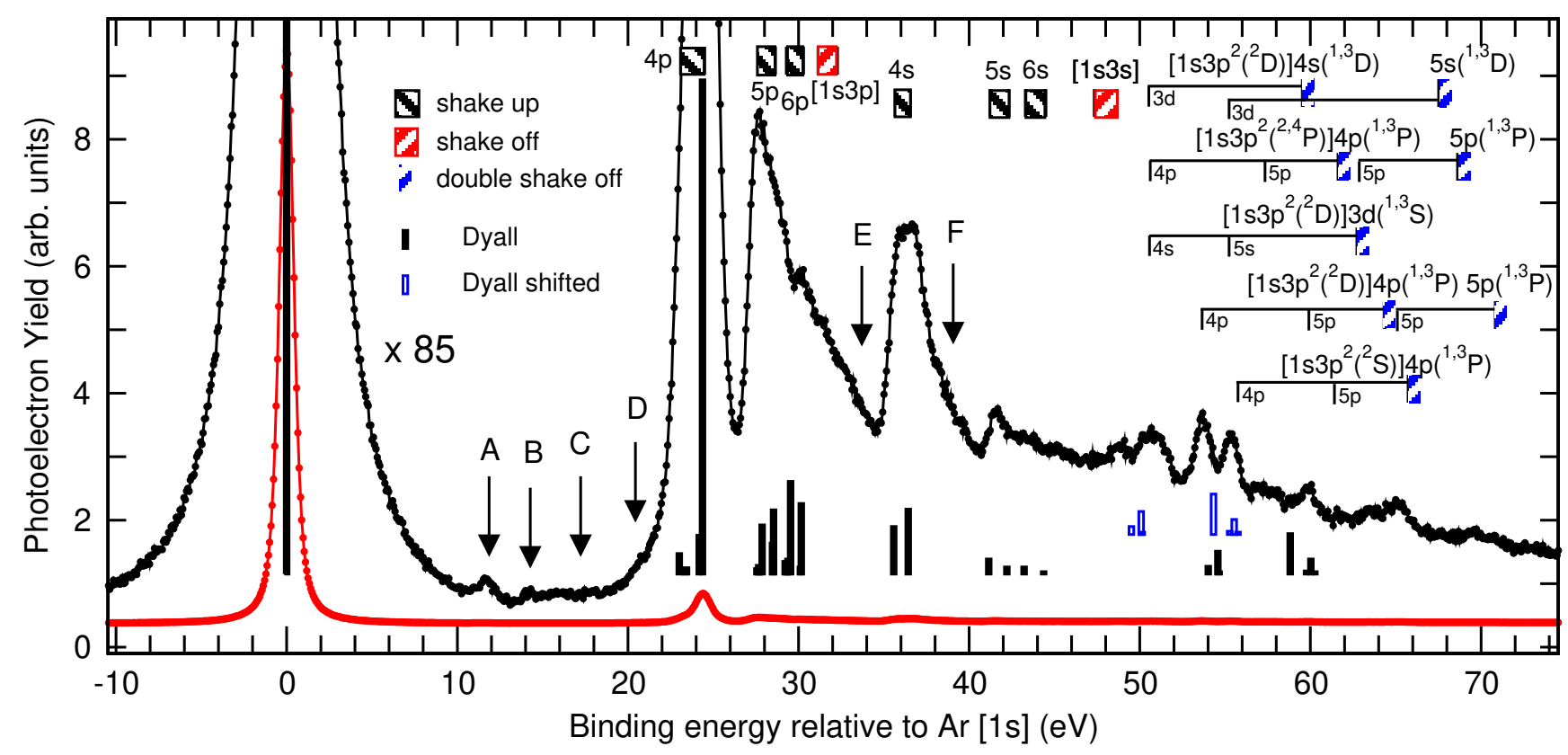

FIG. 2: (color online) The Ar $[1 s]$ photoelectron spectrum including the region of the valence orbital shake-up structures (relative binding energies of 20 to $48 \mathrm{eV}$ ) and double shake-up structures (above $48 \mathrm{eV}$ ) measured using a photon energy of $h \nu=3900 \mathrm{eV}$. For the black line the intensity axis is stretched by a factor of 85 . The vertical bars indicate the energy positions and intensities as calculated by Dyall [31]. The assignments of the single shake-up states are indicated by boxes that show the spread of the states belonging to a given configuration. The red boxes filled diagonally from bottom left to top right and labeled by $[1 s 3 p]$ and $[1 s 3 s]$ indicate the double ionization thresholds populated via shake off. The black boxes filled diagonally from top left to bottom right labeled $n^{\prime} s^{\prime}$ or $n^{\prime} p^{\prime}$ indicate the corresponding single shake-up states. The spectral features labeled $\mathrm{A}$ to $\mathrm{F}$ are discussed in the text. In the double shake-up region above $48 \mathrm{eV}$ the estimated positions for the double shake-up states and the corresponding ionization thresholds are indicated by the vertical bar diagrams above the spectrum. The labels above the horizontal lines of the type $\left[1 s 3 p^{2}\left({ }^{S} L\right)\right] n^{\prime} l^{\prime}\left(S^{\prime} L^{\prime}\right)$ specify the double-ionization thresholds, which are represented with diagonal blue lines from bottom left to up right. The labels next to the small vertical lines of the type $n^{\prime} l^{\prime}$ specify the second excited electron. The hollow blue bars below the spectrum indicate the theoretical predictions of Dyall for the double shake transitions, however, shifted to lower energies by $4.5 \mathrm{eV}$. 
showing also double shake-up transitions of the type $\left[1 s 3 p^{2}\right] n_{1}^{\prime} l_{1}^{\prime} n_{2}^{\prime} l_{2}^{\prime}$; note that the lower indices 1 and 2 describe the two excited electrons. The spectrum can be divided in three regions, namely the Ar $[1 s]$ main line located at a binding energy of 3206.3(3) eV as well as the single shake-up region at relative binding energies of 20 to $48 \mathrm{eV}$ and the double shake-up region with relative binding energies above the latter value.

The calculations of Dyall [31] (black vertical bars in Fig. 2) take only direct shake transitions into account. In these transitions the $1 s$ electron interacts with the photon and is promoted into the continuum while a $3 s$ or $3 p$ electron is excited via shake into a $n^{\prime} s^{\prime}$ or $n^{\prime} p^{\prime}$ orbital. These direct shake transitions are described by the matrix element $\left|\left\langle n l \mid n^{\prime} l^{\prime}\right\rangle\right|^{2}$. As mentioned above, only monopole transitions, i.e. $l=l^{\prime}$, are allowed based on the matrix element. Moreover, this matrix element leads only to a ${ }^{2} S$ symmetry for the final ionic state, which is strictly valid in pure $L S$-coupling. However, Dyall took also relativistic effects into account, which lead to a configuration mixing and weak spectral contributions caused by final ionic states with symmetries different from ${ }^{2} S$. These final ionic states are important in the discussion of the knock-down Auger decays (type 3), see further below.

The assignment for the single-shake transition resulting from the work of Dyall [31] is indicated in the upper part of the spectrum by boxes filled with diagonal lines. The energy positions for the $[1 s 3 s]$ and $[1 s 3 p]$ thresholds are estimated by using the $Z+1$ approximation, i.e. the ionization thresholds of K II [34], as well as the theoretical spin-orbit and exchange splittings for corresponding thresholds in argon [31]. In addition to the calculated shake satellites, the spectrum shows six small spectral features indicated with A to F, which are not reproduced by the theoretical work of Dyall [31]. Structures A to D are previously explained in Ref. [8]. In detail, structures A to C originate from energy losses of the $\mathrm{Ar}[1 s]$ photoelectron that lead to interatomic processes, namely $3 p \rightarrow 4 s, 3 p \rightarrow 5 s$, and $3 p \rightarrow n s$ transitions with $n \geq 6$ in a neutral argon atom. Based on calculations, Southworth et al. assigned shoulder D to $[1 s 3 p] 3 d$ and $[1 s 3 p] 4 s$ shake transitions [8].

A second type of shake process is the conjugate shake process. In this case the photon promotes the $1 s$ electron in a $n^{\prime} p^{\prime}$ orbital, accompanied by a monopole shake of a $3 s$ or $3 p$ electron into the $\epsilon s, p$ continuum. Shoulder $\mathrm{F}$ is assigned as such a transition. It is located at the high-energy side of the $[1 s 3 s] 4 s$ transitions and possesses a binding energy which is 39 $\mathrm{eV}$ higher than that of the $[1 s]$ level. In detail, we assign it to the $[1 s 3 s] 4 p$ conjugate shake transitions since its term value of $\cong 9 \mathrm{eV}$ relative to the $[1 s 3 s]$ threshold agrees well with 
the term value of $\cong 8 \mathrm{eV}$ for the $[1 s 3 p] 4 p$ shake transitions relative to the $[1 s 3 p]$ threshold.

The energy region indicated by $\mathrm{E}$ is between the exchange and spin-orbit split $\left[1 s 3 p\left({ }^{1} P,{ }^{3} P_{2,1,0}\right)\right]$ thresholds and the lowest $[1 s 3 s] 4 s$ transitions so that the strong decrease of the photoionization cross section cannot be explained by single shake-up states. A similar spectral feature is observed for the shake region of neon and explained by the shake-off continuum $[35,36]$, i.e. by a double ionization with the emission of a fast photoelectron and a very slow shake-off electron. We assume that this is also valid for argon.

As shown in Figure 2, the most relevant excited initial states for the $K L L$ Auger spectrum are the singly ionized states $[1 s 3 p] 4 p$ and $[1 s 3 p] 5 p$ as well as the doubly ionized states $[1 s 3 p]$. The latter ones are due to shake-off transitions and are shown to represent $\cong 20 \%$ of the intensity of the $[1 s]$ main line $[32,33]$.

In the double shake-up region above $48 \mathrm{eV}$ relative binding energy there is no obvious agreement with the predictions by Dyall [31]. In order to obtain an approach to the assignment we indicated with the diagonal blue lines from bottom left to up right the expected energy positions for a number of double ionization thresholds. Above the corresponding horizontal lines the thresholds are labeled by $\left[1 s 3 p^{2}\right] n^{\prime} l^{\prime}\left(S^{\prime} L^{\prime}\right)$. The labels next to the small vertical lines of the type $n^{\prime} l^{\prime}$ specify the second excited electron.

The thresholds $\left[1 s 3 p^{2}\left({ }^{2} D\right)\right] 4 s, 5 s\left({ }^{1,3} D\right),\left[1 s 3 p^{2}\left({ }^{2} D\right)\right] 3 d\left({ }^{1,3} S\right)$, and $\left[1 s 3 p^{2}\left({ }^{4} P\right)\right] 4 p$ are estimated based on the $Z+1$ approximation, i.e. we assume that their binding energy relative to the $[1 s]$ line is identical to the respective states in $\mathrm{K} I I I$ relative to the ground state of K II; the corresponding energy values are taken from NIST [34]. The remaining thresholds are estimated based on the thresholds obtained from the $Z+1$ approximation and the theoretical splittings between the $\left[1 s 3 p^{2}\right] n^{\prime} l^{\prime}$ obtained by Dyall [31].

Using the indicated thresholds, the different $\left[1 s 3 p^{2}\right] 4 p^{2}$ satellites calculated by Dyall [31] possess term values of $\cong 8 \mathrm{eV}$, i.e. comparable to the values for the $[1 s 3 p] 4 p$ satellites. However, it has to be expected that a $4 \mathrm{p}$ electron screens another $4 \mathrm{p}$ electron less from the atomic core than a $3 p$ electron. Consequently, we expect larger $4 p$ term values for the $\left[1 s 3 p^{2}\right] 4 p^{2}$ than for the $[1 s 3 p] 4 p$ configuration. From this we conclude that the calculated energy positions for the double shake-up states are too high in energy.

By shifting the calculated values for the double shake satellites by $4.5 \mathrm{eV}$ towards lower energies (hollow vertical bars) a reasonable agreement with the spectral features is reached. Based on this finding, we tentatively assign the broad peak at $\cong 51 \mathrm{eV}$ rel- 


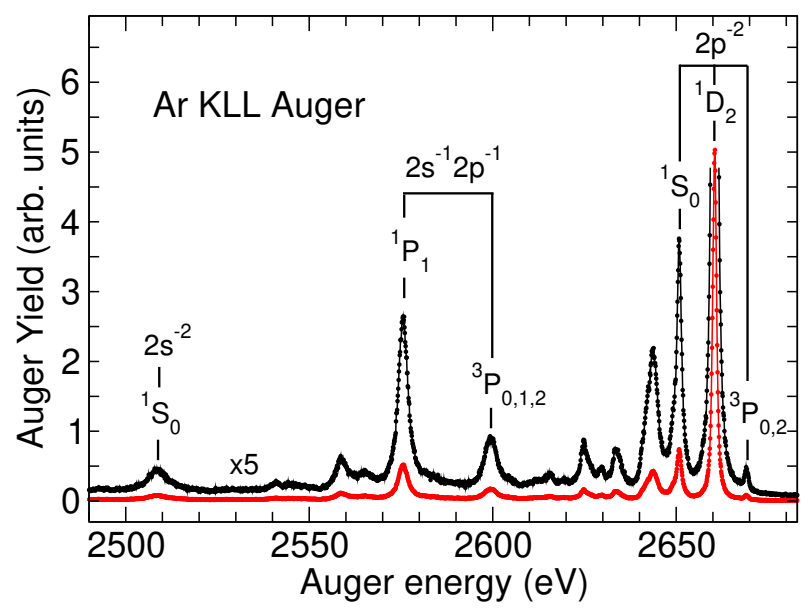

FIG. 3: (color online) The KLL Auger spectra measured subsequent to photoionization with a photon energy of $h \nu=4500 \mathrm{eV}$. The diagram lines are indicated by the vertical-bar diagrams above the spectrum while all other spectral features are due to shake structures.

ative binding energy to the $\left[1 s 3 p^{2}\left({ }^{2} D\right)\right] 4 s\left({ }^{1} D\right) 3 d\left({ }^{2} S_{1 / 2}\right),\left[1 s 3 p^{2}\left({ }^{2} D\right)\right] 4 s\left({ }^{3} D\right) 3 d\left({ }^{2} S_{1 / 2}\right)$, and $\left[1 s 3 p^{2}\left({ }^{4} P\right)\right] 4 p^{2}\left({ }^{1} D\right)\left({ }^{4} P_{1 / 2}\right)$ satellites, the peak at $\cong 53.5 \mathrm{eV}$ to $\left[1 s 3 p^{2}\left({ }^{2} D\right)\right] 4 p^{2}\left({ }^{1} D\right)\left({ }^{2} S_{1 / 2}\right)$ satellite, as well as the peak at $\cong 55.5 \mathrm{eV}$ to the $\left[1 s 3 p^{2}\left({ }^{2} D\right)\right] 5 s\left({ }^{1} D\right) 3 d\left({ }^{2} S_{1 / 2}\right)$, $\left[1 s 3 p^{2}\left({ }^{2} D\right)\right] 5 s\left({ }^{3} D\right) 3 d\left({ }^{2} S_{1 / 2}\right)$, and $\left[1 s 3 p^{2}\left({ }^{2} S\right)\right] 4 p^{2}\left({ }^{1} S\right)\left({ }^{2} S_{1 / 2}\right)$ satellites. The respective assignments are indicated by small vertical bars in the upper part of the figure. Based on this assignment as well as the indicated thresholds we assigned the remaining spectral features to $\left[1 s 3 p^{2}\right] 4 p 5 p$ and $\left[1 s 3 p^{2}\right] 5 p^{2}$ double shake satellites, see also small vertical bars above the spectrum.

\section{THE Ar $K L L$ AUGER DECAY}

Figure 3 shows the overview spectrum of the KLL Auger transitions measured using a photon energy of $h \nu=4500 \mathrm{eV}$, i.e. the spectrum contains all possible shake processes. In this figure the different diagram lines (type 1) are indicated by vertical-bar diagrams above the spectrum. All lines not indicated represent the shake structures which are the main target of the present investigation. For this study we restrict the spectrum to the Auger energy range from $2605 \mathrm{eV}$ to $2655 \mathrm{eV}$ with the $[1 s] \rightarrow\left[2 p^{2}\right]$ Auger transitions, see Fig. 4, because of two different reasons. First, previous studies on $\mathrm{H}_{2} \mathrm{~S}$ [20] showed that each diagram line possesses the same shake structure. Second, the linewidths of the $[1 s] \rightarrow\left[2 s^{2}\right]$ 


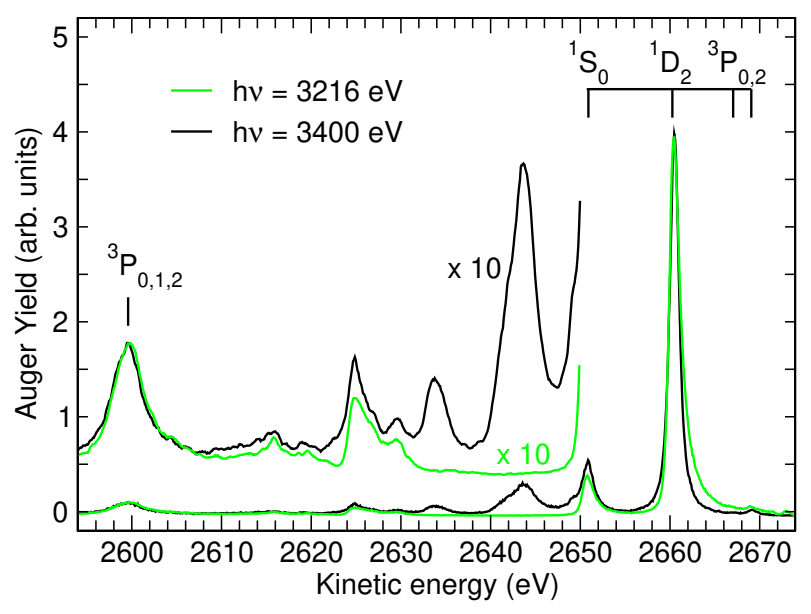

FIG. 4: (color online) The $K L L$ Auger spectra in the region of the $[1 s] \rightarrow\left[2 p^{2}\left({ }^{1} S_{0},{ }^{1} D_{2},{ }^{3} P_{0,2}\right)\right]$ transitions and the $[1 s] \rightarrow\left[2 s 2 p\left({ }^{3} P_{0,1,2}\right)\right]$ diagram lines measured subsequent to photoionization with a photon energy of $h \nu=3216 \mathrm{eV}$ (green) and $h \nu=3400 \mathrm{eV}$ (black). The diagram lines are indicated by vertical bars above the spectra. For reasons of presentation, the green spectrum measured at $h \nu=3216 \mathrm{eV}$ is shifted by $0.55 \mathrm{eV}$ to lower energies to compensate the PCI shift. For the same reasons, the spectra multiplied by 10 are smoothed by averaging over 5 neighboring points.

and the $[1 s] \rightarrow[2 s 2 p]$ Auger transitions are larger due to the shorter lifetime of the $2 s$ hole so that the strongly overlapping shake transitions cannot be resolved.

Based on the $\operatorname{Ar}[1 s]$ photoelectron spectrum previously discussed we selected two different photon energies, namely $h \nu=3216 \mathrm{eV}$ and $h \nu=3400 \mathrm{eV}$, for the ionization process of the $K L L$ Auger spectra used in the data analysis. The photon energy $h \nu=3216 \mathrm{eV}$ was selected in order to exclude contributions from the Auger decays of shake-up structures in the photoelectron spectrum (types 4 to 6 ) while at $h \nu=3400 \mathrm{eV}$ the shake-up contributions are expected to be fully developed. The spectra measured with photon energies of $h \nu=3216$ $\mathrm{eV}$ and $h \nu=3400 \mathrm{eV}$ are presented in Fig. 4 in green and black, respectively, and show remarkable differences. The energy positions of the $\operatorname{Ar}[1 s] \rightarrow\left[2 p^{2}\left({ }^{1} S_{0},{ }^{1} D_{2},{ }^{3} P_{0,2}\right)\right]$ and Ar $[1 s] \rightarrow\left[2 s 2 p\left({ }^{3} P_{0,1,2}\right)\right]$ diagram lines are indicated by the vertical bars above the spectrum. In case of the spectrum measured at an ionization energy of $h \nu=3216 \mathrm{eV}$ only the $\mathrm{Ar}[1 s]$ state can be populated so that all lines but the diagram in this spectrum are due to shake processes during the Auger decay (type 2).

The spectrum measured subsequent to a photoionization energy of $h \nu=3400 \mathrm{eV}$ shows 
additional significant spectral features, mainly in the kinetic energy region from $2630 \mathrm{eV}$ to $2650 \mathrm{eV}$ which are assigned to the Auger spectra of the satellite states observable in the photoelectron spectrum, i.e. types 4 to 6 .

For reasons of presentation, the green spectrum measured at $h \nu=3216 \mathrm{eV}$ is shifted by $0.55 \mathrm{eV}$ to lower energies to compensate the post-collision interaction (PCI) shift. Moreover, the $\operatorname{Ar}[1 s] \rightarrow\left[2 p^{2}\left({ }^{1} S_{0},{ }^{1} D_{2}\right)\right]$ transitions show a significant PCI distortion. In contrast to this, such a modification of the lineshape is practically absent for the $\operatorname{Ar}[1 s] \rightarrow\left[2 s 2 p\left({ }^{3} P_{0,1,2}\right)\right]$ lines measured at the same photon energy. These differences are explained by the fact that the lineshapes are formed by PCI-lineshapes with a width of $655 \mathrm{meV}$ [21] due to the $\mathrm{Ar}[1 s]$ lifetime, convoluted with a Lorentzian of the lifetime widths of the final states [37]. This final-state lifetime width amounts for the $\left[2 p^{2}\right]$ states to $323(15) \mathrm{meV}[22]$ and for the $[2 s 2 p]$ states to $2.17(2) \mathrm{eV}$, see below; the convolution with a Lorentzian of the latter value masks the PCI distortion almost completely. The influence of post-collision interaction for argon is discussed in detail in $[37,38]$.

In the following we shall first discuss in subsection IV A the shake-up processes during the Auger decay (type 2), based on the spectrum measured at $h \nu=3216 \mathrm{eV}$. Then we will discuss in subsection IV B the Auger decays of the shake-up lines in the photoelectron spectrum which are only present in the spectrum recorded using a photon energy of $h \nu=$ $3400 \mathrm{eV}$, namely types 4 to 6 . Moreover, we present in subsection IV C the Ar $[1 s] \rightarrow$ $\left[2 s 2 p\left({ }^{3} P\right)\right]$ diagram line including the splitting into the final states $\left({ }^{3} P_{2,1,0}\right)$. Finally, the $\operatorname{Ar}$ $[2 s]$ photoelectron as well as the $\operatorname{Ar}[1 s] \rightarrow\left[2 s^{2}\right]$ and $\operatorname{Ar}[1 s] \rightarrow[2 s 2 p]$ transitions are fitted in order to determine the lifetime broadenings of the $\operatorname{Ar}[2 s]$ single core hole as well as the $\operatorname{Ar}\left[2 s^{2}\right]$ and $[2 s 2 p]$ double core holes, see subsection IV D.

\section{A. The $\mathbf{A r}[1 s] \rightarrow\left[2 p^{2}\left({ }^{1} S_{0},{ }^{1} D_{2},{ }^{3} P_{0,2}\right) 3 p\right] n^{\prime \prime}\left(p^{\prime \prime}, d^{\prime \prime}\right)$ Auger shake-up transitions}

In this subsection we discuss the shake transitions during the Auger decay (type 2). For this purpose Figure 5 shows the corresponding $K L L$ Auger spectrum measured subsequent to a photon energy of $h \nu=3216 \mathrm{eV}$ together with two different calculations, which are used to support the assignment of the shake-up transitions. The relativistic calculations shown in part b) are performed by including different configurations. The black, red, and blue spectra show the results by including only the $\left[2 p^{2} 3 p\right] 4 p$ configuration, the configurations $\left[2 p^{2} 3 p\right] 4 p$ 


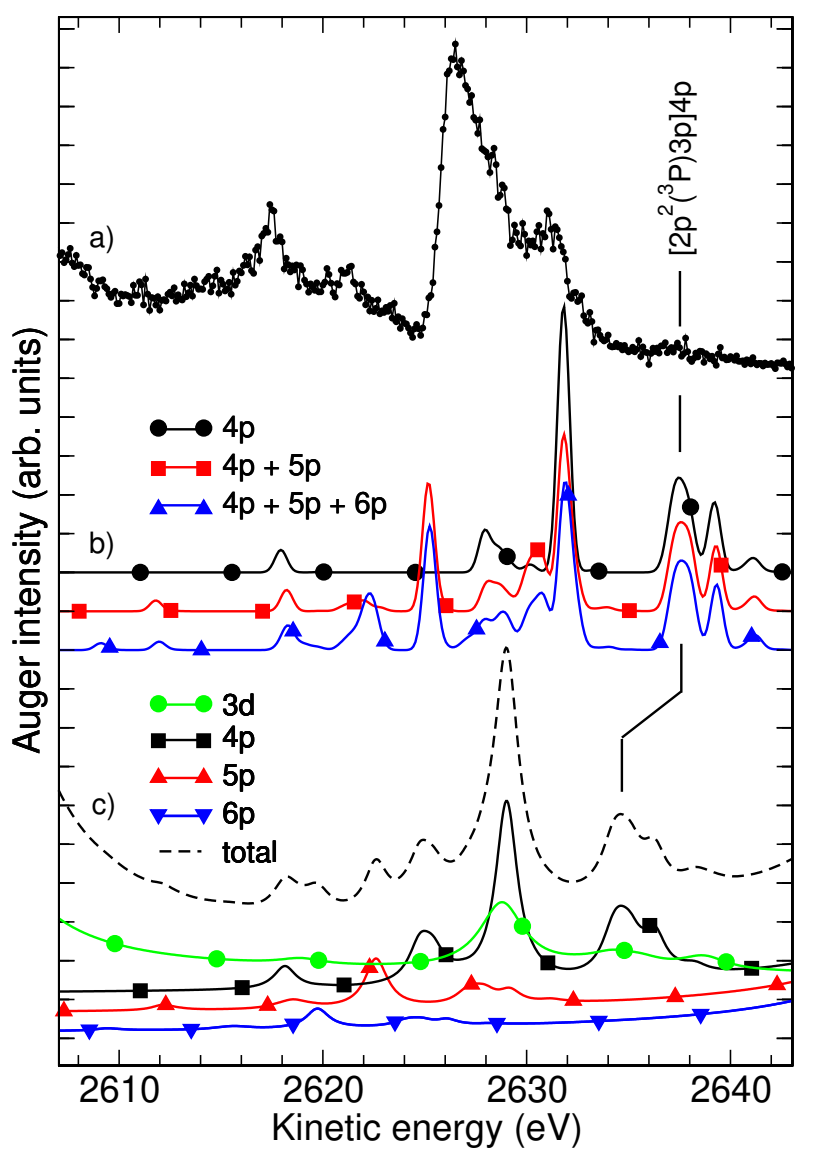

FIG. 5: (color online) The region of the $\operatorname{Ar}[1 s] \rightarrow\left[2 p^{2}\left({ }^{1} S_{0},{ }^{1} D_{2},{ }^{3} P_{0,2}\right) 3 p\right] n(p, d)$ Auger shake-up transitions: a) The experimental results. b) Relativistic calculations. The black line marked by cirlces shows the calculations including only the $\left[2 p^{2} 3 p\right] 4 p$ configuration. The red (blue) line marked by squares (up triangles) displays the results of the calculations taking the configurations $\left[2 p^{2} 3 p\right] 4 p$ and $\left[2 p^{2} 3 p\right] 5 p\left(\left[2 p^{2} 3 p\right] 4 p,\left[2 p^{2} 3 p\right] 5 p\right.$, and $\left.\left[2 p^{2} 3 p\right] 6 p\right)$ into account. c) non-relativistic calculations. Shown are the contributions of the configurations $\left[2 p^{2} 3 p\right] 3 d$ (solid green line marked by circles), $\left[2 p^{2} 3 p\right] 4 p$ (solid black line marked by squares), $\left[2 p^{2} 3 p\right] 5 p$ (solid red line marked by up triangles), and $\left[2 p^{2} 3 p\right] 6 p$ (solid blue line marked by down triangles) as well as the total spectrum (black dashed line). Note that the baselines of the contributions in b) and c) are shifted relative to each other.

and $\left[2 p^{2} 3 p\right] 5 p$, as well as the configurations $\left[2 p^{2} 3 p\right] 4 p,\left[2 p^{2} 3 p\right] 5 p$, and $\left[2 p^{2} 3 p\right] 6 p$, respectively. The non-relativistic calculations shown in part c) include the configurations $\left[2 p^{2} 3 p\right] 3 d$ (solid green line), $\left[2 p^{2} 3 p\right] 4 p$ (solid black line), $\left[2 p^{2} 3 p\right] 5 p$ (solid red line), and $\left[2 p^{2} 3 p\right] 6 p$ (solid blue line), as well as $\left[2 p^{2} 3 p\right] 4 s$ and $\left[2 p^{2} 3 p\right] 4 d$. The latter two configurations show only minor contributions and are not shown in Fig. 5 c) for clarity reasons. 
Both calculations show differences in the absolute intensities and energy positions and resemble only qualitatively the experimental Auger spectrum. This prevents a detailed assignment of the shake structures based on the calculations. However, they suggest that the configurations $\left[2 p^{2} 3 p\right] 3 d,\left[2 p^{2} 3 p\right] 4 p,\left[2 p^{2} 3 p\right] 5 p$, and $\left[2 p^{2} 3 p\right] 6 p$ have to be taken into account.

In order to achieve an approach to the assignment of the observed transitions, a combined approach consisting of a fit analysis, the two calculations and the $\mathrm{Z}+2$ model is utilized. In the following we shall first describe our fit model and the result of the fit analysis. After this we shall assign the observed spectral features using our calculations and the Z+2 model.

In the fit approach, only transitions to the $\left[2 p^{2}\left({ }^{1} S_{0},{ }^{1} D_{2}\right) 3 p\right] n^{\prime \prime}\left(p^{\prime \prime}, d^{\prime \prime}\right)$ final states were taken into account. The transitions to the $\left[2 p^{2}\left({ }^{3} P_{0,2}\right) 3 p\right] n^{\prime \prime}\left(p^{\prime \prime}, d^{\prime \prime}\right)$ states were neglected since even the most intense ones of this type, namely the $\left[2 p^{2}\left({ }^{3} P_{0,2}\right) 3 p\right] 4 p$, see Fig. 5 a), are hardly visible in the experimental results. In a first fit approach identical energy splittings between the $\operatorname{Ar}[1 s] \rightarrow\left[2 p^{2}\left({ }^{1} S_{0},{ }^{1} D_{2}\right)\right]$ diagram lines and the $\operatorname{Ar}[1 s] \rightarrow\left[2 p^{2}\left({ }^{1} S_{0},{ }^{1} D_{2}\right) 3 p\right] n^{\prime \prime}\left(p^{\prime \prime}, d^{\prime \prime}\right)$ shake transitions were assumed, i.e. a coupling of the $3 p$ or $3 d$ electron to the $\left[2 p^{2}\left({ }^{1} S_{0}\right.\right.$, $\left.{ }^{1} D_{2}\right)$ ] states was neglected. Since this approach did not result in a satisfactory description of the spectrum, in the next step coupling between the $\left[2 p^{2}\right]$ double core hole and the $[3 p]$ valence hole was included. In this way we arrived to the states $\left[2 p^{2}\left({ }^{1} S_{0}\right) 3 p\left({ }^{2} P\right)\right]$ and $\left[2 p^{2}\left({ }^{1} D_{0}\right) 3 p\left({ }^{2} P,{ }^{2} D,{ }^{2} F\right)\right]$, i.e. four-fold splitting. For the shake transitions spin flips were assumed to be very small so that quartet states were neglected. The spin-orbit interaction of the ${ }^{2} L$ states to the components $J=L+1 / 2$ and $J=L-1 / 2$ were also neglected since the resulting splittings were calculated to be in the order of 0.2 to $0.5 \mathrm{eV}$ depending on the individual states $\left[2 p^{2}\left({ }^{1} S_{0}\right) 3 p\left({ }^{2} P\right)\right]$ and $\left[2 p^{2}\left({ }^{1} D_{2}\right) 3 p\left({ }^{2} P,{ }^{2} D,{ }^{2} F\right)\right]$. Note that these splittings agree well with the spin-orbit splitting of the $3 p^{-1}$ state in the $\mathrm{Z}+2$ atom Ca IV of $\cong 385 \mathrm{meV}$ [34]. These splittings are significantly smaller than the lifetime broadening $\Gamma=\Gamma_{[1 s]}+\Gamma_{\left[2 p^{2}\right]}=655 \mathrm{meV}+323 \mathrm{meV}=978 \mathrm{meV}[21,22]$, justifying an omission. As for lifetime broadenings, the same values as for the main lines were applied.

Fig. 6 shows the fit result of the Auger spectrum in the region of the shake transitions which go along with the $[1 s] \rightarrow\left[2 p^{2}\left({ }^{1} S_{0},{ }^{1} D_{2}\right)\right]$ diagram line; the corresponding spectrum is recorded using a photon energy of $\mathrm{h} \nu=3216 \mathrm{eV}$. The red solid line through the data points represents the fit result and the black dashed line the background.

In detail, the background consists of a step at $\cong 2615 \mathrm{eV}$ as well as two broad Gaussians at $\cong 2637 \mathrm{eV}$ and at $\cong 2645 \mathrm{eV}$. The step at $\cong 2615 \mathrm{eV}$ takes into account the 


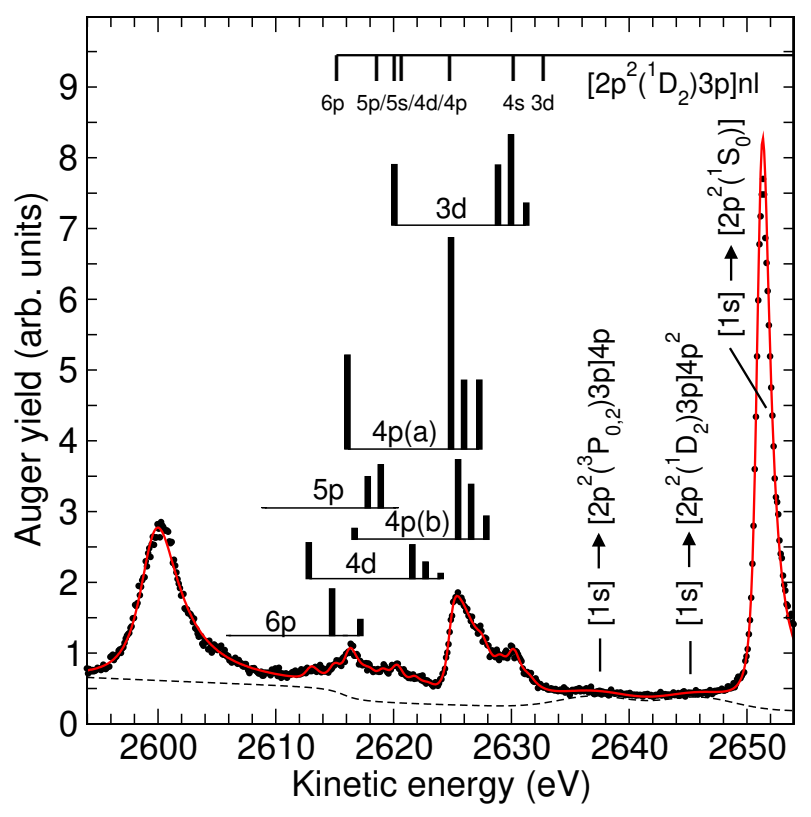

FIG. 6: (color online) Fit result of the Ar $[1 s] \rightarrow\left[2 p^{2}\left({ }^{1} S_{0},{ }^{1} D_{2}\right)\right]$ Auger spectrum in the region of the shake transitions; this spectrum is recorded with a photon energy $\mathrm{h} \nu=3216 \mathrm{eV}$. The solid red line through the data points represents the fit result and the dashed black line the background. The six vertical-bar diagrams indicate the energy positions and relative intensities of the observed shake-up Auger transitions. The vertical bar diagram in the upper part of the spectrum indicates the energy positions of the $[1 s] \rightarrow\left[2 p^{2}\left({ }^{1} D_{2}\right) 3 p\right] n^{\prime \prime} l^{\prime \prime}$ shake-up transitions using the $\mathrm{Z}+2$ approximation as well as the energy position of the $[1 s] \rightarrow\left[2 p^{2}\left({ }^{1} D_{2}\right)\right]$ diagram line as a reference.

$[1 s] \rightarrow\left[2 p^{2}\left({ }^{1} D_{2}\right) 3 p\right] \epsilon l$ shake-off transitions. The first broad Gaussian at $\cong 2637 \mathrm{eV}$ is assigned to the weak $[1 s] \rightarrow\left[2 p^{2}\left({ }^{3} P_{2,0}\right) 3 p\right] 4 p$ transitions. This assignment is based on the present calculations, see Fig. 5, which predict such transitions in this energy range, however, with strongly overestimated intensities. The second broad Gaussian at $\cong 2645 \mathrm{eV}$ is, based on energy arguments, preliminarily assigned to a recapture process of the type $[1 s] \epsilon p \rightarrow\left[2 p^{2}\left({ }^{1} D_{2}\right) 3 p\right] 4 p^{2}$, i.e. a recapture of the photoelectron during the Auger decay. This preliminary assignment is based on energy arguments since at the used photon energy of $\mathrm{h} \nu=3216 \mathrm{eV}$ the energy position of the $[1 s] \epsilon p \rightarrow\left[2 p^{2}\left({ }^{1} D_{2}\right)\right] 4 p$ is found $\cong 19 \mathrm{eV}$ above the $[1 s] \rightarrow\left[2 p^{2}\left({ }^{1} D_{2}\right)\right]$ diagram line [37]. The same splitting is observed between the $[1 s] \rightarrow\left[2 p^{2}\left({ }^{3} P_{2,0}\right) 3 p\right] 4 p$ transitions observed at $2626 \mathrm{eV}$, see below, and the discussed broad Gaussian feature at $2645 \mathrm{eV}$. At $\cong 2651 \mathrm{eV}$ the intense $[1 s] \rightarrow\left[2 p^{2}\left({ }^{1} S_{0}\right)\right]$ diagram line can be seen. 
The six vertical-bar diagrams shown in Fig. 6 indicate the main results of the fit analysis, namely the energy positions and intensities of the shake-up transitions during the Auger decay. From the fit analysis we obtained a splitting of 8.7(1), 9.9(1) and 11.7(3) $\mathrm{eV}$ between the transitions to final states with the parent states $\left[2 p^{2}\left({ }^{1} S_{0}\right) 3 p\left({ }^{2} P\right)\right]$ and $\left[2 p^{2}\left({ }^{1} D_{2}\right) 3 p\left({ }^{2} P,{ }^{2} D,{ }^{2} F\right)\right]$. These values agree well with the results of the present relativistic calculations of $8.5,10.0$, and $13.2 \mathrm{eV}$ and indicate that the applied fit approach describes the essential parts of the physics involved. From the fit analysis we also derive that the intensity of the shakes during the Auger decay amounts 4.8(1.0)\% of the total Auger intensity. This value is based on the assumption that the shake probability is independent from the $\left[2(s, p)^{2}\right]$ final state.

In the next step we shall assign the shake transitions. The six vertical-bar diagrams show that not only monopole shake transitions (i.e. $3 p \rightarrow n^{\prime \prime} p^{\prime \prime}$ transitions where both electrons couple to $\left.{ }^{1} S\right)$ contribute to the spectrum since only three of such transitions $(n=$ $4,5,6)$ are expected in this energy region. To assign the observed transitions we utilize the above given calculation which suggest that the configurations $\left[2 p^{2} 3 p\right] 3 d,\left[2 p^{2} 3 p\right] 4 p,\left[2 p^{2} 3 p\right] 5 p$, and $\left[2 p^{2} 3 p\right] 6 p$ have to be taken into account. As mentioned above we employ the $\mathrm{Z}+2$ approximation, i.e. the energy values of excitations in Ca III [34], to estimate the energy positions of the final states $\left[2 p^{2}\left({ }^{1} D_{2}\right) 3 p\right] n^{\prime \prime} l^{\prime \prime}$; these values are given by the vertical-bar diagram in the upper part of the figure. Based on these data we assign the obtained finalstate configuration to $\left[2 p^{2} 3 p\right] 3 d,\left[2 p^{2} 3 p\right] 4 p,\left[2 p^{2} 3 p\right] 4 d,\left[2 p^{2} 3 p\right] 5 p$, and $\left[2 p^{2} 3 p\right] 6 p$ as indicated in Fig. 6. The two multiplets labeled $4 p(a)$ and $4 p(b)$ are split by $\cong 750 \mathrm{meV}$ and are both assigned to the $\left[2 p^{2} 3 p\right] 4 p$ due to the small splitting and the large intensity.

The suggested additional splitting of $\left[2 p^{2} 3 p\right] 4 p$ configuration can be understood by assuming monopole shake transitions. In this case the $3 p$ hole and the $4 p$ electron couple to a ${ }^{1} S$. As a result, the final states have to have either ${ }^{1} S$ or ${ }^{1} D$ symmetry, based on the coupling of the two $2 p$ holes. By taking the spin-orbit interaction due to the $3 p$ hole into account one obtains in LS coupling the final states $\left[2 p^{2}\left({ }^{1} S_{0}\right) 3 p\left({ }^{2} P_{1 / 2,3 / 2}\right)\right] 4 p\left({ }^{1} S_{0}\right)$ and $\left[2 p^{2}\left({ }^{1} D_{2}\right) 3 p\left({ }^{2} P_{1 / 2,3 / 2},{ }^{2} D_{3 / 2,5 / 2},{ }^{2} F_{5 / 2,7 / 2}\right)\right] 4 p\left({ }^{1} D_{2}\right)$, i.e. 2 final states belonging to $\left[2 p^{2}\left({ }^{1} S_{0}\right)\right]$ and six final states belonging to $\left[2 p^{2}\left({ }^{1} D_{2}\right)\right]$. Generally, only half of these states are observed, since spin-orbit interaction is too low to be resolved, see above. However, due to the spacial vicinity of the $3 p$ hole and the $4 p$ electron the interaction is expected to be stronger than for higher- $n^{\prime \prime}\left[2 p^{2} 3 p\right] n^{\prime \prime} p^{\prime \prime}$ configuration. This can lead to a splitting between e.g. the 
$\left[2 p^{2}\left({ }^{1} D_{2}\right) 3 p\left({ }^{2} P_{1 / 2,3 / 2}\right)\right] 4 p\left({ }^{1} D_{2}\right)$ states, which is larger than the spin-orbit splitting between the $\left[2 p^{2}\left({ }^{1} D_{2}\right) 3 p\left({ }^{2} P_{1 / 2,3 / 2}\right)\right]$ parent states and can explain the observed splitting. For higher $n^{\prime \prime}$ the described effect is too small and only the splittings of the parent states are observable.

As already mentioned above, the given assignment including the final-state configurations $\left[2 p^{2} 3 p\right] 3 d$ and $\left[2 p^{2} 3 p\right] 4 d$ demonstrates that also non-monopole shake transitions contribute to the spectrum. As a consequence, other non-monopole transitions like Ar $[1 s] \rightarrow\left[2 p^{2} 3 p\right] n^{\prime \prime} p^{\prime \prime}$ with $3 p$ and $n^{\prime \prime} p^{\prime \prime}$ coupling to a symmetry different from ${ }^{1} S$ cannot be excluded. As for the photoelectron spectrum, see above, such non-monopole shake transitions can be explained by configuration interaction of the ionic states caused by relativistic effects. Finally we want to point out that in the present fit analysis we observe a large number of overlapping lines so that the intensity information given by the vertical bars is not very reliable. In particular, the energy positions of the transitions $[1 s] \rightarrow\left[2 p^{2}\left({ }^{1} S_{0}\right) 3 p\left({ }^{2} P\right)\right] 3 d$ and $[1 s] \rightarrow\left[2 p^{2}\left({ }^{1} D_{2}\right) 3 p\left({ }^{2} F\right)\right] 5 p$ overlap strongly in our fit analysis. Based on this analysis the spectral intensity is assigned to the first transition, although in reality it is probably shared by both transitions. In summary, we give a coherent assignment based on calculations and the Z+2 approximation. However, for an ultimate assignment much more sophisticated calculations have to be performed.

B. The Auger decays of the Ar $[1 s 3(s, p)] n^{\prime} l^{\prime}$ shake-up and $[1 s 3(s, p)]$ shake-off states

In this subsection we focus on the Auger decays of the $\operatorname{Ar}[1 s 3(s, p)] n^{\prime} l^{\prime}$ shake-up and $[1 s 3(s, p)]$ shake-off states. In a first step we shall discuss the region of the shake-up and spectator decays during the Auger process (types 4 to 6), which is shown in Fig. 7. Further below we will discuss the $\operatorname{Ar}[1 s 3(s, p)] n^{\prime} l^{\prime} \rightarrow\left[2 p^{2}\left({ }^{1} D\right)\right]$ knock-down Auger transitions (type 3), which are shown in Fig. 8; they contribute by roughly $0.5 \%$ to the Auger decays of the shake-up satellites.

The spectrum shown in Fig. 7 is fitted with the same approach as the shake-transitions during the Auger decay, see above. The result of this fit approach is given by the red solid line through the data points, while the background is indicated by the dashed black line. As the main result of the fit analysis, five vertical-bar diagrams indicate the energy positions and intensities of the observed transitions. 


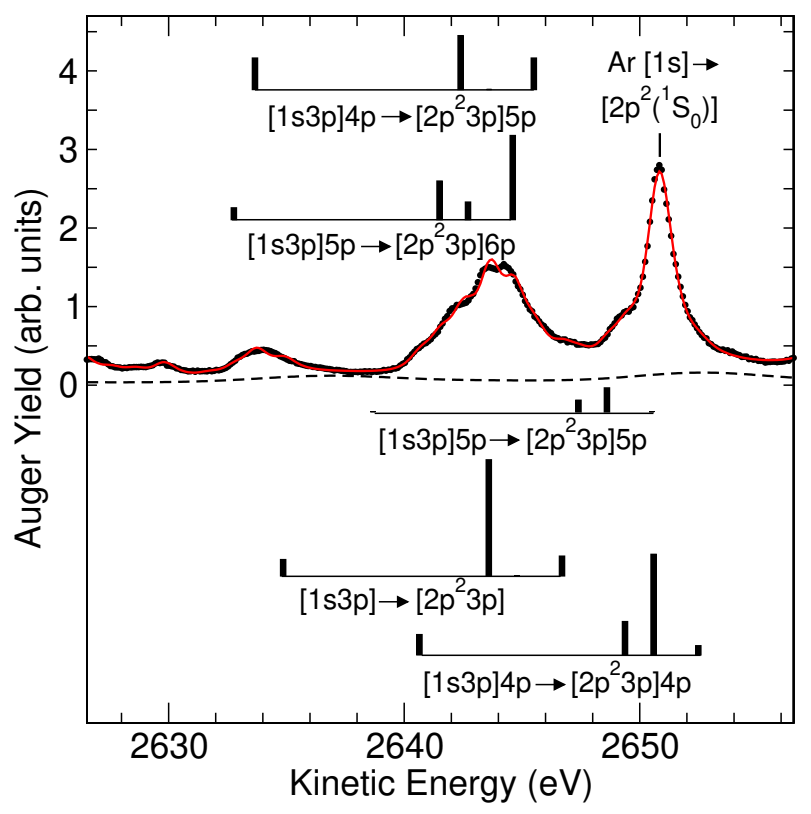

FIG. 7: (color online) Fit result of the Ar KLL Auger spectrum in the region of the Auger transitions of the $\operatorname{Ar}[1 s 3 p] n^{\prime} l^{\prime}$ photoelectron shake satellites to the $\left[2 p^{2}\left({ }^{1} S_{0},{ }^{1} D_{2}\right)\right]$ double core hole; this spectrum is recorded with a photon energy $\mathrm{h} \nu=3400 \mathrm{eV}$. The solid red line through the data points represents the fit result and the dashed black line the background. The five vertical-bar diagrams indicate the energy positions and relative intensities of the observed Auger transitions.

To assign these transitions only the $\operatorname{Ar}[1 s 3 p] 4 p,[1 s 3 p] 5 p$ and $[1 s 3 p]$ initial states are taken into account. The first two initial states are the most intense singly ionized satellite states as can be seen in the photoelectron spectrum, see Fig. 2. The doubly ionized [1s3p] initial state caused by shake-off transitions is characterized by the emission of two electrons and cannot be observed in the present single-channel measurements. However, theoretical and experimental studies suggest that the $[1 s 3 p]$ shake-off state has $\cong 20 \%$ of the intensity of the $[1 s]$ main line $[32,33]$ so that it has to be taken into account. For the initial states Ar $[1 s 3 p] 4 p$ and $[1 s 3 p] 5 p$ spectator (type 4) and shake-up (type 5) Auger decays to the final states $\left[2 p^{2} 3 p\right] 4 p,\left[2 p^{2} 3 p\right] 5 p$, and $\left[2 p^{2} 3 p\right] 6 p$ are taken into account. The energy positions of the transitions are estimated based on the energy positions of the $[1 s 3 p] n^{\prime} p^{\prime}$ initial states taken from Fig. 2 and the $\left[2 p^{2} 3 p\right] n^{\prime \prime} l^{\prime \prime}$ final states taken from Fig. 6. Finally, the energy position of the $[1 s 3 p] \rightarrow\left[2 p^{2} 3 p\right]$ transition (type 6 ) is estimated based on the $\mathrm{Z}+1$ approximation for the core-hole state and the $\mathrm{Z}+2$ approximation for the final state, giving a kinetic energy of $\cong 19 \mathrm{eV}$ below the one of the corresponding $[1 s] \rightarrow\left[2 p^{2}\right]$ diagram line. In this way we 


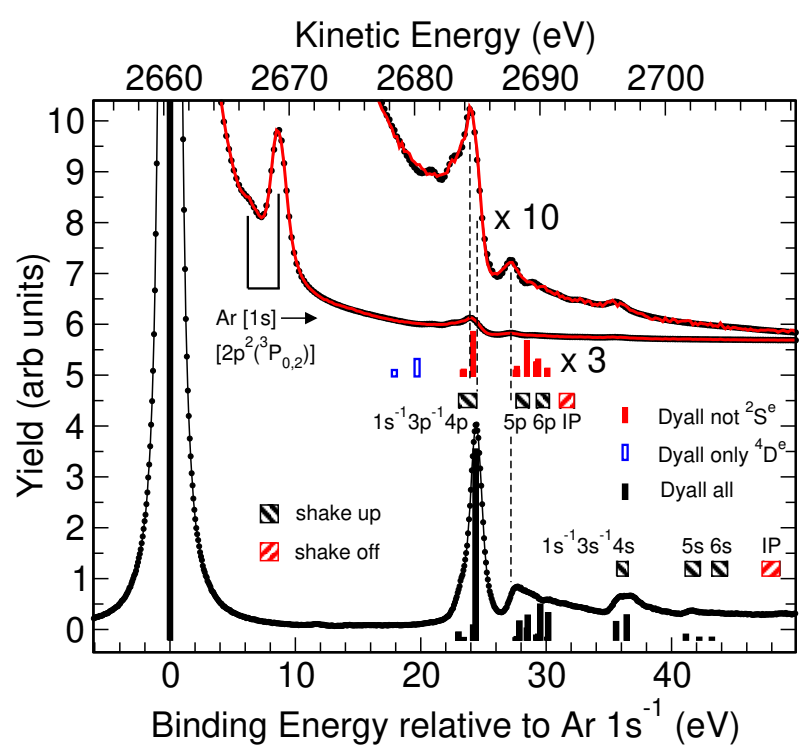

FIG. 8: (color online) The upper part shows the region of the $[1 s 3(s, p)] n^{\prime} l^{\prime} \rightarrow\left[2 p^{2}\left({ }^{1} D_{2}\right)\right]$ transitions. The red solid line through the data points represents the fit result. The lower part indicates the photoelectron spectrum with the corresponding $[1 s 3(s, p)] n^{\prime} l^{\prime}$ shake satellites. The black filled vertical bars below the photoelectron spectrum indicate the intensities and energy positions of the shake satellites as calculated by Dyall [31]. The energy positions of the different $[1 s 3(s, p)] n^{\prime} l^{\prime}$ states as well as $[1 s 3(s, p)]$ double ionization thresholds are indicated by the boxes above the spectrum. The red filled vertical bars below the Auger spectrum indicate the results of the calculations of Dyall, however limited to states with symmetries different from ${ }^{2} S^{e}$ and intensity multiplied by a factor of three. Finally, the blue hollow vertical bars at relative binding energies $\cong 19 \mathrm{eV}$ indicate the theoretical results of states with ${ }^{4} D^{e}$ symmetry alone and the $[1 s]$ main line aligned to the $[1 s] \rightarrow\left[2 p^{2}\left({ }^{1} S_{0}\right)\right]$ Auger transition. For more details, see text.

obtain the assignment given below the various vertical-bar diagrams in Fig. 8.

Fig. 4 exhibits also differences between the spectra recorded with the photon energies $h \nu=3216 \mathrm{eV}$ and $3400 \mathrm{eV}$ in the energy region from $2610 \mathrm{eV}$ to $2630 \mathrm{eV}$. The spectrum measured at the latter photon energy shows generally higher intensities than the former one. These differences are probably due to decays of higher- $n^{\prime}[1 s 3 p] n^{\prime} p^{\prime}$ or $[1 s 3 s] n^{\prime} s^{\prime}$ states as well as shake transitions like $[1 s 3 p] 4 p \rightarrow\left[2 p^{2} 3 p\right] 6 p$, which are not taken into account in our analysis due to strong overlapping with the shakes during the Auger decay (type 2).

Finally we shall discuss the Auger decay of the $[1 s 3(s, p)] n^{\prime} l^{\prime}$ shake-up states to the $\left[2 p^{2}\right]$ configuration, i.e. transitions where the excited electron returns to its original shell 
(type 3 ). The relevant energy range is at kinetic energies above the $[1 s] \rightarrow\left[2 p^{2}\right]$ transitions and can be seen in the upper part of Figure 8. For comparison, the lower part of the figure shows the $[1 s 3 s] n^{\prime} s^{\prime}$ and $[1 s 3 p] n^{\prime} p^{\prime}$ satellite region of the photoelectron spectrum with the $[1 s]$ main line aligned to the $[1 s] \rightarrow\left[2 p^{2}\left({ }^{1} D\right)\right]$ diagram line in the Auger spectrum. The similarities in the photoelectron and the Auger spectra clearly support the assignment of the Auger structures to $[1 s 3(s, p)] n^{\prime} l^{\prime} \rightarrow\left[2 p^{2}\left({ }^{1} D\right)\right]$ Auger transitions. Note that the background for the Auger spectrum is much more curved than in the photoelectron spectrum. This curvature is predominately due to the high-energy tail of the Lorentzian lineshape of the $[1 s] \rightarrow\left[2 p^{2}\left({ }^{1} D\right)\right]$ transition and indicates that the intensity ratio of the $[1 s 3(s, p)] n^{\prime} l^{\prime} \rightarrow\left[2 p^{2}\right]$ transitions and the $[1 s] \rightarrow\left[2 p^{2}\left({ }^{1} D\right)\right]$ diagram line in the Auger spectrum is much smaller than the intensity ratio of the $[1 s 3(s, p)] n^{\prime} l^{\prime}$ shake-up states and the $[1 s]$ main photoelectron line.

Although the structures in the photoelectron and the Auger spectra are quite similar, a detailed comparison reveals interesting differences. First, we shall point out that we do not observe at $\cong 2674 \mathrm{eV}$ the $\left[2 p^{2}\left({ }^{1} S\right)\right]$ counterpart to the $[1 s 3 p] 4 p \rightarrow\left[2 p^{2}\left({ }^{1} D\right)\right]$ transitions. One should expect to see this transition if one assumes the same intensity ratio for $[1 s 3 p] 4 p \rightarrow\left[2 p^{2}\left({ }^{1} S\right)\right]$ to $[1 s 3 p] 4 p \rightarrow\left[2 p^{2}\left({ }^{1} D\right)\right]$ as for the diagram lines $[1 s] \rightarrow\left[2 p^{2}\left({ }^{1} S\right)\right]$ and $[1 s] \rightarrow\left[2 p^{2}\left({ }^{1} D\right)\right]$ of about 1:10. Obviously, the intensity depends on the diagram line and is not a process of the valence shell alone. Second, the peak maxima in the Auger and the photoelectron spectrum differ in particular for the $[1 s 3 p] 4 p$ and $[1 s 3 p] 5 p$ shake-up satellites by about $600 \mathrm{meV}$ as indicated by the vertical dashed lines; this value is about one order of magnitude larger than the maximum expected error caused by aligning the two spectra to each other. This peak shift can be explained by a significant reduction of the intensity of the ${ }^{2} S_{1 / 2}$ states, see below. Third, the exact spectral structure shows differences. For example, there is a clear minimum in the Auger spectrum at $\cong 2688.5 \mathrm{eV}$ which is missing in the photoelectron spectrum. Moreover, the lineshapes of the $[1 s 3 p] 4 p$ and $[1 s 3 s] 4 s$ clearly change. For the first peak the low-energy shoulder becomes more pronounced and for the second peak the high-energy component disappears completely. Since each spectral feature consists of different components, as can be seen by the black vertical bars below the photoelectron spectrum, we can conclude that the individual $[1 s 3(s, p)] n^{\prime} l^{\prime}$ shake-up states show different probabilities to decay to the $\left[2 p^{2}\right]$ configuration.

In the following we shall discuss the reason for the individual decay probabilities for 
the $[1 s 3(s, p)] n^{\prime} l^{\prime}$ states. For this reason, the black filled vertical bars below the spectrum indicate the calculated intensities and energy positions for all $\operatorname{Ar}$ [1s] shake satellites [31] and show generally a good agreement between experiment and theory regarding both intensities and energy positions. In addition, the red filled vertical bars between the photoelectron and the Auger electron spectrum show the theoretical results for the states which have total symmetries different from ${ }^{2} S_{1 / 2}$. Their intensities are multiplied by a factor of three as compared to the black filled vertical bars and they describe reasonably well the main spectral features of the Auger spectrum; this includes the peak shift to lower energy for both first peaks as discussed below. This result suggests that for the decay to the $\left[2 p^{2}\right]$ configuration shake-up states with a symmetry different from ${ }^{2} S_{1 / 2}$ are preferred.

From the observations we suggest as most efficient decay mechanism to the $\left[2 p^{2}\right]$ configuration a knock-down process, i.e. a direct interaction between the Auger electron leaving the $2 p$ shell and the excited electron in the valence shell. In this way, the excited electron can exchange energy and momentum and jump back to its original $3 s$ or $3 p$ shell. In this context we point out that all calculated shake-up satellites in the photoelectron spectrum have even parity. The same holds for the final $\left[2 p^{2}\right]$ configuration so that the outgoing Auger electron is only allowed to possess $\epsilon s$ or $\epsilon d$ character. Moreover, the Auger electron has to carry away the difference in angular momentum between the core-hole and the Auger final state. In case of a $\left[2 p^{2}\left({ }^{1} D\right)\right]$ final state and an $\epsilon s$ Auger electron this is possible only for core-hole states with $D$-symmetry while for an $\epsilon d$ Auger electron the symmetries $S, P, D, F$ and $G$ are possible. However, for core-hole photoelectron satellites with $S$ symmetry, the hole in the valence shell and the excited electron couple to zero. Since they also have to couple to an angular momentum of zero in the Auger final state due to the $3 p^{6}$ valence shell, the valence shell cannot accept angular momentum from the outgoing Auger electron. As a result, the $\left[2 p^{2}\right]$ configuration can be reached only via a shake-down process which is described by the overlap matrix elements $\left\langle 3 s^{++} \mid n s^{+}\right\rangle$and $\left\langle 3 p^{++} \mid n p^{+}\right\rangle$with ++ , indicating the orbitals in the dicationic Auger final state and + in the core-ionized state; these matrix elements are expected to be relatively small and explain the low intensities of the ${ }^{2} S_{1 / 2}$ states in the Auger spectrum. In contrast, for the calculated [1s] shake satellites with $P$ or $D$ symmetry the Auger electron can accept angular momentum and, as a consequence energy, more efficiently from the shaken valence electron so that the latter one can jump back to its original shell. 
For the Auger final state $\left[2 p^{2}\left({ }^{1} S\right)\right]$ and $\epsilon s$ Auger electron the core-hole state has to be of $S$ symmetry and in case of a $\epsilon d$ Auger electron of $D$ symmetry. Core-hole satellite states with $P$ symmetry cannot decay in LS-coupling to the $\left[2 p^{2}\left({ }^{1} S\right)\right]$ without violating either parity or angular-momentum conservation; they are allowed only due to spin-orbit coupling and, therefore, are very weak. This situation is comparable with the $\left[2 p^{2}\left({ }^{3} P\right)\right]$ diagram line, which is also very weak as compared to the $\left[2 p^{2}\left({ }^{1} D\right)\right]$ diagram line due to the same reason [10].

Since the core-hole states with $S$ symmetry cannot exchange angular momentum with the Auger electron, see above, they are expected to be weak in the discussed type of Auger decay. This explains, together with the branching ratio for the decay to the $\left[2 p^{2}\left({ }^{1} S\right)\right]$ and the $\left[2 p^{2}\left({ }^{1} D\right)\right]$ state of about 1:10 that they are absent in the Auger spectrum. Contrary to this, core-hole satellites with $D$ symmetry are allowed to exchange energy and angular momentum with the outgoing Auger electron so that they can decay to the $\left[2 p^{2}\left({ }^{1} S\right)\right]$ Auger final state. Their expected energy positions based on the calculations of Dyall [31] and the splitting of the diagram lines (see Table I) are indicated with blue hollow bars below the Auger spectrum. The more intense line agrees quite well in energy with the spectral feature at $\cong 2681 \mathrm{eV}$ so that we tentatively assign the latter feature to a $[1 s 3 p] n^{\prime} l^{\prime}\left(D_{1 / 2}\right) \rightarrow\left[2 p^{2}\left({ }^{1} S\right)\right]$ transition.

In summary, in this subsection we presented the Auger transitions of the shake-up and shake-off states visible in the Ar $[1 s]$ photoelectron spectrum. The transitions are assigned mainly based on the argument of energy positions. Finally, weak $\operatorname{Ar}[1 s 3(s, p)] n^{\prime} l^{\prime} \rightarrow$ $\left[2 p^{2}\left({ }^{1} D\right)\right]$ are observed and explained with the knock-down mechanism.

\section{The Ar $[1 s] \rightarrow\left[2 s 2 p\left({ }^{3} P_{2,1,0}\right)\right]$ diagram lines}

Fig. 9 shows the region of the $\operatorname{Ar}[1 s] \rightarrow\left[2 s 2 p\left({ }^{3} P_{0,1,2}\right)\right]$ diagram lines (type 1$)$ measured subsequent to photoionization with a photon energy of $h \nu=3400 \mathrm{eV}$. This part of the spectrum also includes some $\operatorname{Ar}[1 s] \rightarrow\left[2 p^{2}\left({ }^{1} S_{0},{ }^{1} D_{2}\right) 3(s, p)\right] n^{\prime \prime} l^{\prime \prime}$ Auger shake-up transitions, see dashed subspectrum. The dominating peak at $2600 \mathrm{eV}$ caused by the $\operatorname{Ar}[1 s] \rightarrow\left[2 s 2 p\left({ }^{3} P\right)\right]$ transition clearly exhibits an asymmetric lineshape which is due to the observation of the splitting of the $\left[2 s 2 p\left({ }^{3} P\right)\right]$ configuration into the final states ${ }^{3} P_{0,1,2}$.

To obtain a better insight into the splitting of the $\left[2 s 2 p\left({ }^{3} P\right)\right]$ states the spectrum was 
TABLE I: Summary of the absolute experimental kinetic energies, $E_{k i n, e x p}$, of the diagram lines (type 1 ) as well as the energies, $E_{\text {rel,exp }}$, relative to the $\operatorname{Ar}[1 s] \rightarrow\left[2 p^{2}\left({ }^{1} D\right)\right]$ diagram line. For comparison, the relative kinetic energies, $E_{\text {rel,theo }}$ obtained by different theoretical studies are also given.

\begin{tabular}{|c|c|c|c|c|c|c|c|c|}
\hline \multirow{2}{*}{\multicolumn{2}{|c|}{ configuration state $E_{k i n, e x p}(\mathrm{eV})$}} & \multicolumn{3}{|c|}{$E_{r e l, \exp }(\mathrm{eV})$} & \multicolumn{4}{|c|}{$E_{\text {rel,theo }}(\mathrm{eV})$} \\
\hline & & present work & Ref. [5] & Ref. [6] & Ref. [5] & Ref. [6] & Ref. [4] & Ref. [1] \\
\hline \multirow[t]{4}{*}[2p^{2}]{} & ${ }^{3} P_{2}$ & $8.66(1)$ & \multirow{2}{*}{8.9} & 8.6 & 8.40 & 8.9 & 8.4 & 8 \\
\hline & ${ }^{3} P_{0}$ & $6.51(5)$ & & 6.4 & 6.40 & 6.4 & 6.4 & 6 \\
\hline & ${ }^{1} D_{2}$ & 0 & 0 & 0 & 0 & 0 & 0 & 0 \\
\hline & ${ }^{1} S_{0}$ & $-9.66(1)$ & -9 & -9.6 & -8.10 & -8.9 & -8.0 & -12 \\
\hline \multirow[t]{4}{*}[2s2p]{} & ${ }^{3} P_{2}$ & $-60.35(10)$ & \multirow{3}{*}{\}-61.1} & \multirow{3}{*}{\}-60.8} & -59.82 & -60.2 & -58.8 & -57 \\
\hline & ${ }^{3} P_{1}$ & $-61.75(20)$ & & & -61.22 & -61.7 & -60.1 & -59 \\
\hline & ${ }^{3} P_{0}$ & $-62.15(20)$ & & & -61.98 & -62.5 & -60.9 & -58 \\
\hline & ${ }^{1} P_{1}$ & $-84.89(1)$ & -84.7 & -84.8 & -85.09 & -86.4 & -84.2 & -82 \\
\hline$\left[2 s^{2}\right]$ & ${ }^{1} S_{0}$ & $-151.95(1)$ & -150.5 & -151.6 & -151.52 & -153.6 & -149.6 & -142 \\
\hline
\end{tabular}

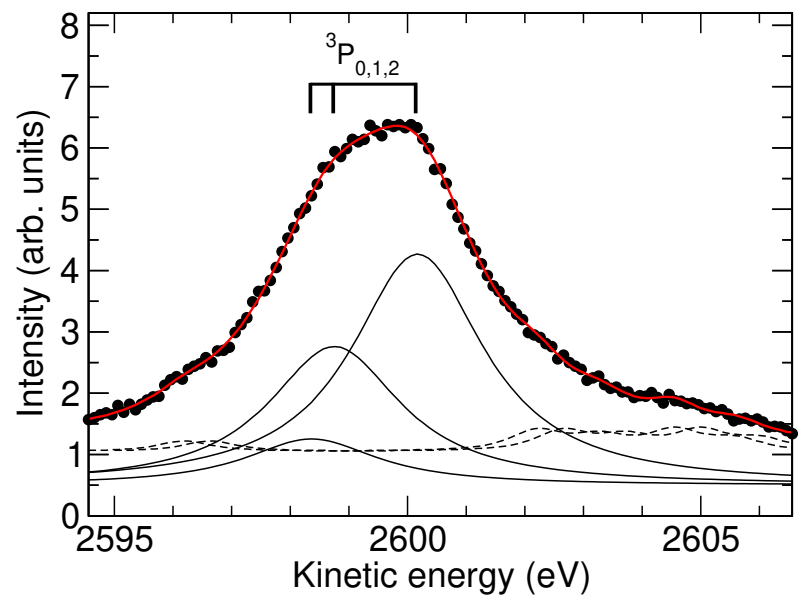

FIG. 9: (color online) The region of the Ar $[1 s] \rightarrow\left[2 s 2 p\left({ }^{3} P_{0,1,2}\right)\right]$ diagram lines. The red solid line through the data points represents the fit result. The black solid subspectra indicate the contributions of the different diagram lines and are assigned by the vertical-bar diagram above the spectrum. The dashed subspectrum indicates the background including some $\operatorname{Ar}[1 s] \rightarrow\left[2 p^{2}\left({ }^{1} S_{0}\right.\right.$, $\left.\left.{ }^{1} D_{2}\right) 3(s, p)\right] n^{\prime \prime} l^{\prime \prime}$ Auger shake-up transitions. 
fitted with 3 broad Lorentzian lines of equal widths representing the three diagram lines, see solid subspectrum. For these lines the intensity ratio was fixed to 1:3:5 according to the statistical weights; this approach is justified by the practically identical intensity ratios obtained by different calculations $[2,5]$. Contrary to this, the energy positions were treated as free parameters. Moreover, a number of narrower lines representing $\operatorname{Ar}[1 s] \rightarrow\left[2 p^{2}\left({ }^{1} S_{0}\right.\right.$, $\left.\left.{ }^{1} D_{2}\right) 3(s, p)\right] n n^{\prime \prime} l^{\prime \prime}$ Auger shake-up transitions were included in the background, see dashed subspectrum. The result of the fit is represented by the red solid line through the data points and yields the kinetic energy positions $2598.8(2) \mathrm{eV}, 2599.2(2) \mathrm{eV}$ and 2600.6(1) eV for the transitions to the final states ${ }^{3} P_{0},{ }^{3} P_{1}$, and ${ }^{3} P_{2}$, respectively. The energy positions of the $\operatorname{Ar}[1 s] \rightarrow\left[2 s 2 p\left({ }^{3} P_{0,1,2}\right)\right]$ diagram lines relative to those of the $\operatorname{Ar}[1 s] \rightarrow\left[2 p^{2}\left({ }^{1} D_{2}\right)\right]$ line are summarized in table I, together with the values for the other diagram lines as obtained from the fit analyses presented above and below. The results of previous experimental and theoretical studies are also given, revealing in particular a good agreement between the present results and the theoretical values of Ref. [5].

\section{Lifetime broadening of Ar $[2 s]$ or $\left[2 s^{2}\right]$ containing states}

In this subsection we investigate the lifetime broadenings of the $\mathrm{SCH}$ state $\mathrm{Ar}[2 s]$ as well as the DCH states $\left[2 s^{2}\left({ }^{1} S_{0}\right)\right],\left[2 s 2 p\left({ }^{1} P_{1}\right)\right]$, and $\left[2 s 2 p\left({ }^{3} P_{0,1,2}\right)\right]$. The latter values are derived from the Auger spectrum by subtracting the well-known Ar [1s] lifetime broadening [21] and are compared with the lifetime broadening of the SCH states $\operatorname{Ar}[2 s]$ and $\operatorname{Ar}[2 p]$. While the lifetime broadening of the $\operatorname{Ar}[2 p]$ core hole is well known from literature [39], the values for the $\operatorname{Ar}[2 s]$ core hole given in literature differ significantly from each other $[40,41]$. Because of this we derived an accurate value from an $\operatorname{Ar}[2 s]$ photoelectron spectrum recorded in the course of this study, see Fig. 10(a). We shall start discussing the Ar [2s] lifetime followed by the lifetimes of the DCH states. For convenience the discussed experimental lifetime widths are summarized in Table II.

In a fit analysis of the $\operatorname{Ar}[2 s]$ spectrum a lifetime broadening of $\Gamma_{[2 s], e x p}=2.033(10) \mathrm{eV}$ is derived, based on a Gaussian broadening of $0.545(10) \mathrm{eV}$ representing the experimental resolution. The latter value was determined from a $\operatorname{Ar}[2 p]$ photoelectron spectrum using a lifetime broadening of $\Gamma_{[2 p], \exp }=0.118(4) \mathrm{eV}$ [39]. This value lies between the experimental value of $\Gamma_{[2 s], \text { exp }}=1.84(20) \mathrm{eV}$ of Mehlhorn [40] and $\Gamma_{[2 s], \text { exp }}=2.25(5) \mathrm{eV}$ of Glans et al. [41]. 

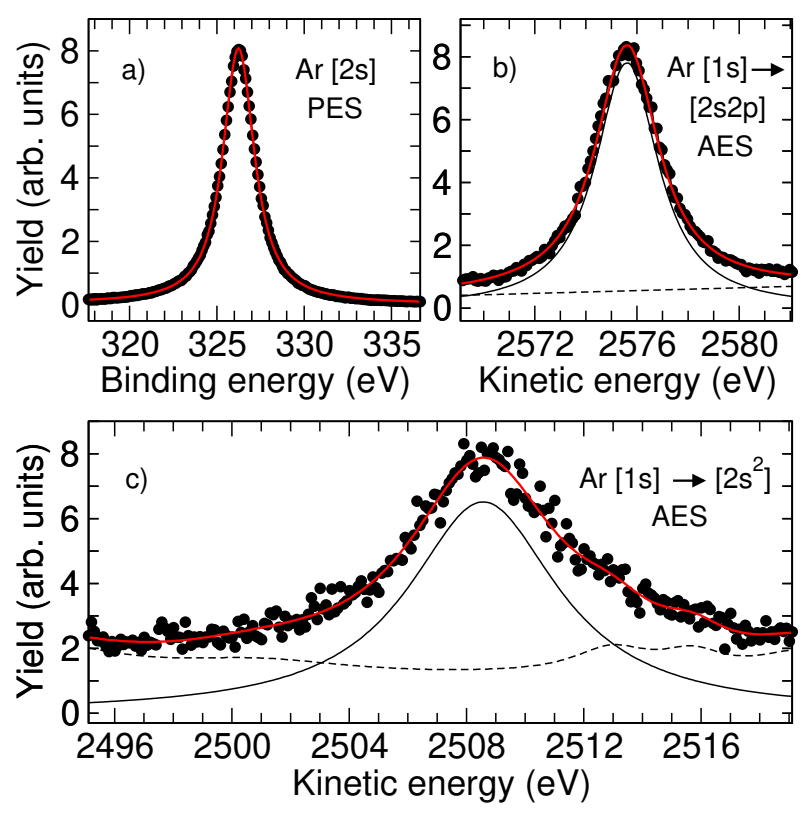

FIG. 10: (color online) The Ar $[2 s]$ photoelectron spectrum (a) as well as the $\operatorname{Ar}[1 s] \rightarrow\left[2 s 2 p\left({ }^{1} P_{1}\right)\right]$ (b) and the $\operatorname{Ar}[1 s] \rightarrow\left[2 s^{2}\left({ }^{1} S_{0}\right)\right]$ Auger transitions. The solid lines through the data points represent the fit result. In case of (b) and (c) the solid subspectrum represents the respective diagram line and the dashed subspectrum the background partially including shake satellites.

The present experimental lifetime broadening for the $[2 s]$ core hole deviates considerably from the theoretical value of $\Gamma_{[2 s], t h e o}=1.63 \mathrm{eV}$ obtained by Krause and Oliver [42]. This deviation for the $[2 s]$ hole is in contrast to the results of Krause and Oliver for the Ar [1s] hole $\left(\Gamma_{[1 s], \exp }=0.655 \mathrm{eV}[21], \Gamma_{[1 s], \text { theo }}=0.680 \mathrm{eV}\right)$ and $\operatorname{Ar}[2 p]$ hole $\left(\Gamma_{[2 p], \exp }=0.118(4)\right.$ $\left.\mathrm{eV}[39], \Gamma_{[2 p], \text { theo }}=0.127 \mathrm{meV}\right)$, where good agreement between experiment and theory was found. A better agreement of the present experimental result of $\Gamma_{[2 s], \exp }=2.033(10)$ is found with the theoretical results of Glans et al. of $\Gamma_{[2 s], t h e o}=1.85 \mathrm{eV}[41]$.

In a fit analysis the Lorentzian width of the $\operatorname{Ar}[1 s] \rightarrow\left[2 s^{2}\left({ }^{1} S_{0}\right)\right]$ diagram line shown in Fig. 10(c) turned out to be $\Gamma=6.15(44) \mathrm{eV}$. This value still contains the Ar $[1 s]$ lifetime so that we obtain $\Gamma_{\left[2 s^{2}\right] \text { exp }}=5.49(44) \mathrm{eV}$ for the $\left[2 s^{2}\left({ }^{1} S_{0}\right)\right]$ DCH state. This results in a ratio of $2.70(22)$ for the lifetimes of the $\left[2 s^{2}\right]$ and the $\left[2 s^{1}\right]$ states, in good agreement with the ratios of $2.76(25)$ for the $\operatorname{Ar}[2 p]$ core-hole states [22], of 2.9(1) for the Ne [1s] core-hole states [23] and confirms for shallow core levels that the DCH lifetime is significantly shorter than twice the SCH lifetime. This observation is a result of the fact that the orbitals energetically above the vacant orbitals experience a higher charge due to the missing electrons so that 
TABLE II: Summary of the experimental argon SCH and DCH lifetime widths $\Gamma_{\text {exp }}$. For comparison with the $\mathrm{DCH}$ lifetimes sums of the corresponding $\mathrm{SCH}$ lifetimes, $\Gamma_{\text {sum }}$, are also given. For the sum values a $\operatorname{Ar}[2 s]$ lifetime width of $\Gamma=2.033(10) \mathrm{eV}$ is used.

\begin{tabular}{lcc}
\hline \hline core hole state & $\Gamma_{\text {exp }}(\mathrm{eV})$ & $\Gamma_{\text {sum }}(\mathrm{eV})$ \\
\hline$[2 p]$ & $0.118(4)^{a}$ & \\
{$[2 s]$} & $2.033(10), 1.84(20)^{b}, 2.25(5)^{c}$ & \\
{$[1 s]$} & $0.665^{d}$ & \\
{$\left[2 p^{2}\right]$} & $0.323(15)^{e}$ & $0.236(8)$ \\
{$\left[2 s 2 p\left({ }^{3} P\right)\right]$} & $2.02(8)$ & \\
{$\left[2 s 2 p\left({ }^{1} P\right)\right]$} & $2.18(2)$ & \\
{$[2 s 2 p]^{f}$} & $2.17(2)$ & $2.151(14)$ \\
{$\left[2 s^{2}\right]$} & $5.49(44)$ & $4.066(20)$ \\
\hline \hline
\end{tabular}

${ }^{a}[39]$

${ }^{b}[40]$

${ }^{c}[41]$

$d[21]$

${ }^{e}[22]$

$f_{\text {weighted average }}$

they shrink and have better overlap with the core hole; this results in a significant increase of the Auger rates. Note that for deeper core holes the ratio is expected to converge to 2 [23]. This is in agreement with the finding of $1.88(25)$ for the I $\left(3 d_{5 / 2}\right)$ level in $\mathrm{CH}_{3} \mathrm{I}$ [43].

In the next step we will investigate the mixed [2s2p] DCH states, which exhibit a different behavior. The $\operatorname{Ar}[1 s] \rightarrow\left[2 s 2 p\left({ }^{3} P_{0,1,2}\right)\right]$ and $\operatorname{Ar}[1 s] \rightarrow\left[2 s 2 p\left({ }^{1} P_{1}\right)\right]$ diagram lines including the fit results are shown in Fig. 9 and Fig. 10(b), respectively. The obtained Lorentzian widths are $\Gamma=2.68(8) \mathrm{eV}$ for the $\operatorname{Ar}[1 s] \rightarrow\left[2 s 2 p\left({ }^{3} P_{0,1,2}\right)\right]$ transition and $\Gamma=$ $2.84(2) \mathrm{eV}$ for the $\operatorname{Ar}[1 s] \rightarrow\left[2 s 2 p\left({ }^{1} P_{1}\right)\right]$ transition. Although the error bars do not allow to derive different lifetimes for the $\left[2 s 2 p\left({ }^{1} P_{1}\right)\right]$ and $\left[2 s 2 p\left({ }^{3} P_{0,1,2}\right)\right]$ states we would like to point out that for the corresponding states of $\mathrm{Br}$ in $\mathrm{HBr}$ such differences have been pinpointed experimentally and theoretically [44]. However, since in the present case the differences are very small and do not influence the discussion, we assume in the following identical lifetimes for the $\left[2 s 2 p\left({ }^{3} P_{0,1,2}\right)\right]$ and $\left[2 s 2 p\left({ }^{1} P_{1}\right)\right]$ core-hole states and obtain as weighted average value 
$\Gamma=2.83(2) \mathrm{eV}$. After subtraction of the Ar $[1 s]$ lifetime broadening a value of $\Gamma_{[2 s 2 p], \exp }=$ $2.17(2) \mathrm{eV}$ is obtained. This lifetime broadening agrees well with the sum of the $[2 s]$ and the $[2 p]$ core hole of $2.151(14) \mathrm{eV}$.

This behavior can be understood based on the dominating decay channel of the Ar $[2 s]$ core hole, namely the $[2 s] \rightarrow[2 p 3 l]$ Coster-Kronig Auger decay which explains the short lifetime of the $2 s$ vacancy. As a result of the additional vacancy in the $2 p$ shell, the numbers of Coster-Kronig Auger decay channels is statistically reduced by a factor of $5 / 6$. By taking this factor into account one obtains a lifetime broadening of $5 / 6 \cdot \Gamma_{[2 s], \exp }+\Gamma_{[2 p], \exp }=1.810 \mathrm{eV}$, i.e. a value which is too small so that the difference between this value and the experimental result of $\Gamma_{[2 s 2 p], \exp }=2.17(2) \mathrm{eV}$ has to be explained by the contraction of the outermost orbitals. A similar effect has been observed for the Ar [1s2p] double core-hole states [24].

In summary, the results on the $[2 s 2 p]$ point out that competing processes influence the lifetime on mixed DCH states, namely the reduction of Auger channels and the increase of the rates of each channel, due to a contraction of the orbitals involved.

\section{SUMMARY AND CONCLUSIONS}

State-of-the-art Argon KLL Auger spectra were presented together with an Ar $1 s^{-1}$ photoelectron spectrum. The latter spectrum allows to investigate in detail the Ar $[1 s 3(s, p)] n^{\prime} l^{\prime}$ photoelectron satellite structures, whose non-radiative decay also contributes to the Auger spectrum. A complete assignment of all satellite structures is performed. Furthermore, six types of different decay pathways are analyzed in great detail. The spectra are measured at photon energies of $h \nu=3216 \mathrm{eV}$ and $3400 \mathrm{eV}$, i.e. below and above the $\operatorname{Ar}[1 s 3(s, p)] n^{\prime} l^{\prime}$ photoelectron satellites. This allows to distinguish between the shake transitions during the Auger decay (type 2) and the Auger transitions of the photoelectron satellites (types 4 to 6 ), since the latter ones are only present in the spectrum measured at $3400 \mathrm{eV}$. While the decays of the photoelectron satellites were assigned in previous studies of the Ar KLL Auger spectrum [5,6], the shake transitions during the Auger decay were assigned before only for $\mathrm{H}_{2} \mathrm{~S}$ [20], so that the present results support that these transitions are a general effect. Moreover, we found that the shakes during the Auger contribute to $4.8(1.0) \%$ to the spectrum of the Ar $[1 s] \rightarrow\left[2 l^{2}\right]$ decays.

For the decay of the photoelectron satellites shake-up and spectator transitions are 
observed. In addition, $\operatorname{Ar}[1 s 3(s, p)] n^{\prime} l^{\prime} \rightarrow\left[2 p^{2}\left({ }^{1} D_{2}\right)\right]$ transitions (type 3 ), i.e. transitions where the $n^{\prime} l^{\prime}$ electron fills the [3l] vacancy, are observed; these we mainly assign to knockdown transitions with an exchange of angular momenta between the excited and the Auger electron.

For the $[1 s] \rightarrow\left[2 s 2 p\left({ }^{3} P_{J}\right)\right]$ transition the different components with $J=2,1,0$ are resolved. Moreover, the lifetime broadenings of the $\mathrm{Ar}[2 s] \mathrm{SCH}$ state and the $\left[2 s^{2}\right]$ as well as $[2 s 2 p] \mathrm{DCH}$ states are determined, confirming previously observed trends for DCH states.

\section{Acknowledgement}

Experiments were performed on the GALAXIES beamline at SOLEIL synchrotron, France. We are grateful to the SOLEIL staff for the smooth operation of the facility. We also gratefully acknowledge significant contributions of Prof. Dr. Dennis W. Lindle in the early stage of this work. The work at the Freie Universität Berlin was supported by the Deutsche Forschungsgemeinschaft under Project No. PU180/6-1. AFL acknowledges the support from CAPES (Coordenação de Aperfeiçoamento de Pessoal de Nível Superior) and CNPq (Conselho Nacional de Desenvolvimento Científico e Tecnológico). P. H. has received funding from the European Unions Horizon 2020 research and innovation programme under the Marie Skłodowska-Curie grant agreement No 764787.

[1] D. A. Shirley, Theory of KLL Auger energies including static relaxation, Phys. Rev. A 7, 1520 (1973), https://doi.org/10.1103/PhysRevA.7.1520.

[2] M. H. Chen and B. Crasemann, K-LL Auger Transition Probabilities for Elements with Low and Intermediate Atomic Numbers, Phys. Rev. A 8, 7 (1973), https://doi.org/10.1103/PhysRevA.8.7.

[3] M. O. Krause Argon KLL Auger Spectrum: A Test of Theory, Phys. Rev. Lett. 34, 633 (1975), https://doi.org/10.1103/PhysRevLett.34.633.

[4] F. P. Larkins, Semi-empirical Auger electron energies. I. General method and K-LL line en- 
ergies, J. Phys. B 9, 47 (1976), https://doi.org/10.1088/0022-3700/9/1/006.

[5] J. Vayrynen, R. N. Sodhi, and R. G. Cavell Energies and intensities of the KLL Auger spectra of $\mathrm{SiH}_{4}, \quad \mathrm{PH}_{3}, \quad \mathrm{HCl}$, and $\mathrm{Ar}, \quad \mathrm{J}$. Chem. Phys. 79, 5329 (1983); http://dx.doi.org/10.1063/1.445695.

[6] L. Asplund, P. Kelfve, B. Blomster, H. Siegbahn, and K. Siegbahn, Argon KLL and KLM Auger electron spectra, Phys. Scr. 16, 268 (1977), https://doi.org/10.1088/0031-8949/16/5$6 / 015$.

[7] T. D. Thomas, Transition from Adiabatic to Sudden Excitation of Core Electrons Phys. Rev. Lett. 52, 417 (1984), https://doi.org/10.1103/PhysRevLett.52.417.

[8] S. H. Southworth, T. LeBrun, Y. Azuma, and K. G. Dyall, Argon KM photoelectron satellites J. Electron Spectros. Relat. Phenom. 94, 33 (1998), https://doi.org/10.1016/S03682048(98)00149-2.

[9] O. Travnikova, M. Patanen, J. Söderström, A. Lindblad, J. J. Kas, F. D. Vila, D. Céolin, T. Marchenko, G. Goldsztejn, R. Guillemin, L. Journel, T. X. Carroll, K. J. Børve, P. Decleva, J. J. Rehr, N. Mårtensson, M. Simon, S. Svensson, and L. J. Sæthre, EnergyDependent Relative Cross Sections in Carbon 1s Photoionization: Separation of Direct Shake and Inelastic Scattering Effects in Single Molecules, J. Phys. Chem. A 123, 7619 (2019), https://doi.org/10.1021/acs.jpca.9b05063.

[10] V. Schmidt, Electron Spectrometry of Atoms using Synchrotron Radiation, (Cambridge University Press, Cambrige, 1997).

[11] K. Faegri and O. Keski-Rahkonen, Sulphur KLL Auger spectra of gaseous sulphur compounds, J. Electron. Spectrosc. Relat. Phenom. 11, 275 (1976), https://doi.org/10.1016/03682048(77)80004-2.

[12] L. Asplund, P. Kelfve, B. Blomster, H. Siegbahn, K. Siegbahn, R. L. Lozes, and U. I. Wahlgren, Molecular Auger electron spectra of second row elements. Sulfur compounds, Phys. Scr. 16, 273 (1977), https://doi.org/10.1088/0031-8949/16/5-6/016.

[13] P. A. Heimann, D. W. Lindle, T. A. Ferrett, S. H. Liu, L. J. Medhurst, M. N. Piancastelli, D. A. Shirley, U. Becker, H. G. Kerkhoff, B. Langer, D. Szostak, and R. Wehlitz, Shake-Off on InnerShell Resonances of Ar, Kr and Xe, J. Phys. B 20, 5005 (1987), https://doi.org/10.1088/0022$3700 / 20 / 19 / 013$.

[14] H. Aksela, S. Aksela, H. Pulkkinen, G. M. Bancroft, and K. H. Tan, Anomalously Strong 
Shake-up Processes in Auger Decay of the Resonantly Excited $2 p^{5} 3 s^{2} 3 p^{6} n l$ States of Ar, Phys. Rev. A 37, 1798(R) (1988), https://doi.org/10.1103/PhysRevA.37.1798.

[15] S. Sundin, F. Kh. Gel'mukhanov, S. J. Osborne, O. Björneholm, A. Ausmees, A. Kikas, S. L. Sorensen, A. Naves de Brito, R. R. T. Marinho, S. Svensson, and H Ågren, Auger Decay of Core-Excited Higher Rydberg States of Carbon Monoxide, J. Phys. B 304267 (1997), https://doi.org/10.1088/0953-4075/30/19/016.

[16] R. Püttner, Y. F. Hu, G. M. Bancroft, A. Kivimäki, M. Jurvansuu, H. Aksela, and S. Aksela, Strong Nonmonopole Shake Transitions in the Br $3 d^{-1} n p \pi(n=6-9)$ Resonant Auger Spectra of HBr, Phys. Rev. A 77, 032705 (2008), https://doi.org/10.1103/PhysRevA.77.032705.

[17] G. Goldsztejn, R. Püttner, L. Journel, R. Guillemin, O. Travnikova, R. K. Kushawaha, B. Cunha de Miranda, I. Ismail, D. Colin, M. N. Piancastelli, M. Simon, and T. Marchenko, Electronic-state-lifetime interference in the hard-x-ray regime: Argon as a showcase Phys. Rev. A 95, 012509 (2017), https://doi.org/10.1103/PhysRevA.95.012509.

[18] T. Kantia, L. Partanen, S. Aksela, and H. Aksela, High Resolution KLL Auger Spectra of Free Sodium and Magnesium Atoms, J. Electron Spectrosc. Relat. Phenom. 180, 58 (2010), https://doi.org/10.1016/j.elspec.2010.04.007.

[19] T. Kantia, S. Aksela, P. Turunen, L. Partanen, and H. Aksela, KLL Auger Decay of Atomic Silicon, J. Phys. B 43, 205002 (2010), https://doi.org/10.1088/0953-4075/43/20/205002.

[20] R. Püttner, D. Céolin, R. Guillemin, R. K. Kushawaha, T. Marchenko, L. Journel, M. N. Piancastelli, and M. Simon, Detailed analysis of shake structures in the KLL Auger spectrum of $H_{2} S$, Phys. Rev. A 93, 042501 (2016), https://doi.org/10.1103/PhysRevA.93.042501.

[21] D. Céolin, J. M. Ablett, D. Prieur, T. Moreno, J. P. Rueff, T. Marchenko, L. Journel, R. Guillemin, B. Pilette, T. Marin, and M. Simon, Hard X-ray photoelectron spectroscopy on the GALAXIES beamline at the SOLEIL synchrotron, J. Electron Spectros. Relat. Phenom. 190, 188 (2013), https://doi.org/10.1016/j.elspec.2013.01.006.

[22] M. Žitnik, R. Püttner, G. Goldsztejn, K. Bučar, M. Kavčič, A. Mihelič, T. Marchenko, R. Guillemin, L. Journel, O. Travnikova, D. Céolin, M. N. Piancastelli, and M. Simon, Twoto-one Auger decay of a double L vacancy in argon, Phys. Rev. A 93, 021401(R) (2016), https://doi.org/10.1103/PhysRevA.93.021401.

[23] G. Goldsztejn, T. Marchenko, R. Püttner, L. Journel, R. Guillemin, S. Carniato, P. Selles, O. Travnikova, D. Céolin, A.F. Lago, R. Feifel, P. Lablanquie, M.N. Pi- 
ancastelli, F. Penent, and M. Simon, Double-Core-Hole States in Neon: Lifetime, PostCollision Interaction, and Spectral Assignment, Phys. Rev. Lett. 117, 133001 (2016), https://doi.org/10.1103/PhysRevLett.117.133001.

[24] R. Püttner, G. Goldsztejn, D. Céolin, J.-P. Rueff, T. Moreno, R.K. Kushawaha, T. Marchenko, R. Guillemin, L. Journel, D.W. Lindle, M. N. Piancastelli, and M. Simon, Direct Observation of Double-Core-Hole Shake-Up States in Photoemission, Phys. Rev. Lett. 114, 093001 (2015), https://doi.org/10.1103/PhysRevLett.114.093001.

[25] M. Simon, R. Püttner, T. Marchenko, R. Guillemin, R. K. Kushawaha, L. Journel, G. Goldsztejn, M. N. Piancastelli, J. M. Ablett, J.-P. Rueff, and D. Céolin, Atomic Auger Doppler effects upon emission of fast photoelectrons, Nat. Commun. 5, 4069 (2014), $10.1038 /$ ncomms5069.

[26] M. Breinig, M. H. Chen, G. E. Ice, F. Parente, B. Crasemann, and G. S. Brown, Atomic Inner-Shell Level Energies Determined by Absorption Spectrometry with Synchrotron Radiation, Phys. Rev. A 22, 520 (1980), https://doi.org/10.1103/PhysRevA.22.520.

[27] M. Alagia, R. Richter, S. Stranges, M. Agåker, M. Ström, J. Söderström, C. Såthe, R. Feifel, S. Sorensen, A. De Fanis, K. Ueda, R. Fink, and J.-E. Rubensson, Core level ionization dynamics in small molecules studied by x-ray-emission threshold-electron coincidence spectroscopy, Phys. Rev. A 71, 012506 (2005), https://doi.org/10.1103/PhysRevA.71.012506.

[28] P. Jönsson, X. He, C. Froese Fischer and I. P. Grant, The grasp2K relativistic atomic structure package, Comput. Phys. Commun. 177, 597 (2007), https://doi.org/10.1016/j.cpc.2007.06.002.

[29] S. Fritzsche, The Ratip program for relativistic calculations of atomic transition, ionization and recombination properties, Comput. Phys. Commun. 183, 1525 (2012), https://doi.org/10.1016/j.cpc.2012.02.016.

[30] M. Hrast, A. Mihelič, K. Bučar, and M. Žitnik, Auger decay of the $2 p$ vacancy in chlorine, Phys. Rev. A 100, 023408 (2019), https://doi.org/10.1103/PhysRevA.100.023408.

[31] K. G. Dyall, Shake theory predictions of excited-state populations following 1s ionisation in argon, J. Phys. B 16, 3137 (1983), https://doi.org/10.1088/0022-3700/16/17/008.

[32] T. A. Carlson and C. W. Nestor, Jr., Calculation of Electron Shake-Off Probabilities as the Result of X-Ray Photoionization of the Rare Gases, Phys. Rev. A 8, 2887 (1973), https://doi.org/10.1103/PhysRevA.8.2887. 
[33] J.-Cl. Dousse and J. Hoszowska, $L$ - and M-shell-electron shake processes following 1 photoionization in argon and krypton, Phys. Rev. A 56, 4517 (1997), https://doi.org/10.1103/PhysRevA.56.4517.

[34] NIST Atomic Level Database, Version 5, http://www.nist.gov/pml/data/asd.cfm

[35] N. Mårtensson and A. Nilsson, High-Resolution Core-Level Photoelectron Spectroscopy of Surfaces and Adsorbates, in Springer Series of Surface Sciences, Vol 35, Application of Synchrotron Radiation, ed. by W. Eberhard (Springer, Berlin, Heidelberg, 1995), pp. 65.

[36] S. Svensson, B. Eriksson, N. Mårtensson, G. Wendin, and U. Gelius, Electron shakeup and correlation satellites and continuum shake-off distributions in X-Ray photoelectron spectra of the rare gas atoms, J. Electron Spectros. Relat. Phenom. 47, 327 (1988), https://doi.org/10.1016/0368-2048(88)85020-5.

[37] R. Guillemin, S. Sheinerman, R. Püttner, T. Marchenko, G. Goldsztejn, L. Journel, R. K. Kushawaha, D. Céolin, M. N. Piancastelli, and M. Simon, Postcollision Interaction Effects in KLL Auger Spectra following Argon 1s Photoionization, Phys. Rev. A 92, 012503 (2015), https://doi.org/10.1103/PhysRevA.92.012503.

[38] R. Huster, W. Sandner, and W. Mehlhorn, Post-Collision Interaction in Inner-Shell Ionisation by Electron Impact: Energy Shift of KLL Auger Electrons of Ne and Ar, J. Phys. B 20, L287 (1986), https://doi.org/10.1088/0022-3700/20/9/004.

[39] M. Jurvansuu, A. Kivimäki, and S. Aksela, Inherent lifetime widths of Ar $2 p^{-1}$, Kr $3 d^{-1}$, Xe $3 d^{-1}$, and Xe $4 d^{-1}$ states, Phys. Rev. A 64, 012502 (2001), https://doi.org/10.1103/PhysRevA.64.012502.

[40] W. Mehlhorn, Das Coster-Kronig- und Auger-Spektrum der $L_{1}$-Schale von Argon Z. Physik 208, 1 (1968), https://doi.org/10.1007/BF01325753

[41] P. Glans, R. E. LaVilla, M. Ohno, S. Svensson, G. Bray, N. Wassdahl, and J. Nordgren Determination of the lifetime width of the argon $L_{1}$-hole state Phys. Rev. A 47, 1539 (1993), https://doi.org/10.1103/PhysRevA.47.1539.

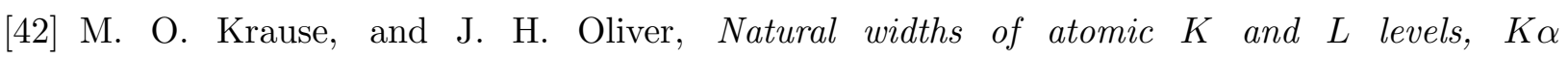
Xray lines and several KLL Auger lines, J. Phys. Chem. Ref. Data 8, 329 (1979), https://doi.org/10.1063/1.555595.

[43] T. Marchenko, G. Goldsztejn, K. Jänkälä, O. Travnikova, L. Journel, R. Guillemin, N. Sisourat, D. Céolin, M. Žitnik, M. Kavčič, K. Bučar, A. Mihelič, B. Cunha de Mi- 
randa, I. Ismail, A.F. Lago, F. Gelmukhanov, R. Püttner, M.N. Piancastelli, and M. Simon, Potential Energy Surface Reconstruction and Lifetime Determination of Molecular Double-Core-Hole States in the Hard X-Ray Regime, Phys. Rev. Lett. 119, 133001 (2017), https://doi.org/10.1103/PhysRevLett.119.133001.

[44] N. Boudjemia, K. Jänkälä, R. Püttner, T. Marchenko, O. Travnikova, R. Guillemin, L. Journel, I. Ismail, D. Koulentianos, S. Kosugi, Y. Azuma, M. Patanen, M. Huttula, D. Céolin, M. N. Piancastelli, and M. Simon, Electron spectroscopy and dynamics of $\mathrm{HBr}$ around the $\mathrm{Br}$ $1 s^{-1}$ threshold, submitted. 